\title{
Differential immune profiles distinguish the mutational subtypes of gastrointestinal stromal tumor
}

\author{
Gerardo A. Vitiello, ${ }^{1}$ Timothy G. Bowler, ${ }^{2}$ Mengyuan Liu, ${ }^{1,3}$ Benjamin D. Medina, ${ }^{1}$ Jennifer Q. Zhang, ${ }^{1}$ Nesteene J. Param, ${ }^{1,3}$ \\ Jennifer K. Loo, ${ }^{1}$ Rachel L. Goldfeder, ${ }^{4}$ Frederic Chibon, ${ }^{5}$ Ferdinand Rossi, ${ }^{1,3}$ Shan Zeng, ${ }^{1}$ and Ronald P. DeMatteo ${ }^{1,3}$ \\ 'Department of Surgery and ${ }^{2}$ Department of Medicine, Memorial Sloan Kettering Cancer Center (MSKCC), New York, New York, USA. ${ }^{3}$ Department of Surgery, University of Pennsylvania, Philadelphia, \\ Pennsylvania, USA. ${ }^{4}$ Cenome Technologies, The Jackson Laboratory for Cenomic Medicine, Farmington, Connecticut, USA. ${ }^{5}$ INSERM U1037, Cancer Research Center of Toulouse, Toulouse, France.
}

\begin{abstract}
Gastrointestinal stromal tumor (GIST) is the most common human sarcoma, frequently characterized by an oncogenic mutation in the KIT or PDCFRA gene. We performed RNA sequencing of 75 human GIST tumors from 75 patients, comprising what we believe to be the largest cohort of CISTs sequenced to date, in order to discover differences in the immune infiltrates of KIT- and PDCFRA-mutant GIST. Through bioinformatics, immunohistochemistry, and flow cytometry, we found that in PDGFRA-mutant GISTs, immune cells were more numerous and had higher cytolytic activity than in KIT-mutant GISTs. PDGFRA-mutant GISTs expressed many chemokines, such as CXCL14, at a significantly higher level when compared with KIT-mutant GISTs and exhibited more diverse driver-derived neoepitope:HLA binding, both of which may contribute to PDCFRA-mutant GIST immunogenicity. Through machine learning, we generated gene expression-based immune profiles capable of differentiating KIT- and PDCFRA-mutant GISTs, and identified additional immune features of high-PD-1and -PD-L1-expressing tumors across all GIST mutational subtypes, which may provide insight into immunotherapeutic opportunities and limitations in GIST.
\end{abstract}

\section{Introduction}

Gastrointestinal stromal tumor (GIST) is the most common sarcoma (1), often originating in the stomach or the small intestine. GIST is a paradigm for targeted molecular therapy, since the majority of GISTs contain an activating mutation in exon 11 of the KIT proto-oncogene, for which there are effective and well-tolerated tyrosine kinase inhibitors $(2,3)$. Imatinib mesylate, which inhibits the KIT oncoprotein, improved median survival in patients with advanced and metastatic GIST from 1 to 5 years (3), while adjuvant imatinib improved recurrence-free survival in patients with resectable disease (4).

PDGFRA-mutant GIST is the second most common form of GIST. Approximately half of PDGFRA-mutant GISTs contain a $D 842 \mathrm{~V}$ substitution, which is inherently resistant to imatinib therapy (5). The majority of PDGFRA-mutant GISTs develop in the stomach $(6,7)$, while KIT-mutant GISTs can arise in the stomach, small intestine, or rectum (8), suggesting an innate and important biologic difference between these driver mutations. Other GIST mutation types also associate with predictable clinicopathologic features. For example, KIT exon 9-mutant GISTs nearly always develop in the small intestine (9); neurofibromin 1-mutant (NF1-mutant) GISTs are more commonly found in the duodenojejunal flexure (10); and succinate dehydrogenase-deficient

Authorship note: GAV and TCB contributed equally to this work. Conflict of interest: The authors have declared that no conflict of interest exists. Copyright: @ 2019, American Society for Clinical Investigation.

Submitted: October 16, 2018; Accepted: February 12, 2019; Published: March 25, 2019. Reference information: J Clin Invest. 2019;129(5):1863-1877. https://doi.org/10.1172/JCl124108.
(SDH-deficient) GISTs are indolent, multifocal, and among the few GISTs - along with those driven by kinase fusions - that metastasize via the lymphatic system $(11,12)$. The underlying biologic mechanisms linking mutation type and clinicopathologic features are not well understood.

Although PDGFRA D842V (D842V)-mutant GISTs do not respond to imatinib, natural history shows that recurrence-free survival is more favorable in patients with any PDGFRA mutation when compared with a KIT mutation (4). This finding, along with the predictable behavior of GIST mutation types, had us hypothesize that the mutational driver may impact other aspects of tumor biology, specifically the tumor microenvironment and host immune response. Our group has extensively characterized the immune infiltrate in Kit-mutant GIST using a genetically engineered mouse model and human specimens (13-17), providing a clear rationale for multiple immunotherapy trials in patients with GIST, but the immune response to PDGFRA-mutant GIST is currently unknown.

In this study, we performed RNA-Seq on 75 surgical specimens from 75 patients with GIST to characterize the immune infiltrates of different GIST mutations. Surprisingly, we discovered that PDGFRA-mutant GIST harbors more immune cells than KIT-mutant GIST; this may be related to oncogene-specific cytokine production or more HLA-diverse neoepitope recognition, suggesting that patients with PDGFRA-mutant GIST might benefit from therapeutic immunomodulation. We trained a random forest machine learning algorithm on RNA-Seq data from all of our KIT- and PDGFRA-mutant GISTs, as well as on a subset of untreated, primary, gastric (UPG) KIT- and PDGFRA-mutant GISTs, in order to characterize a PDGFRA-specific immune land- 


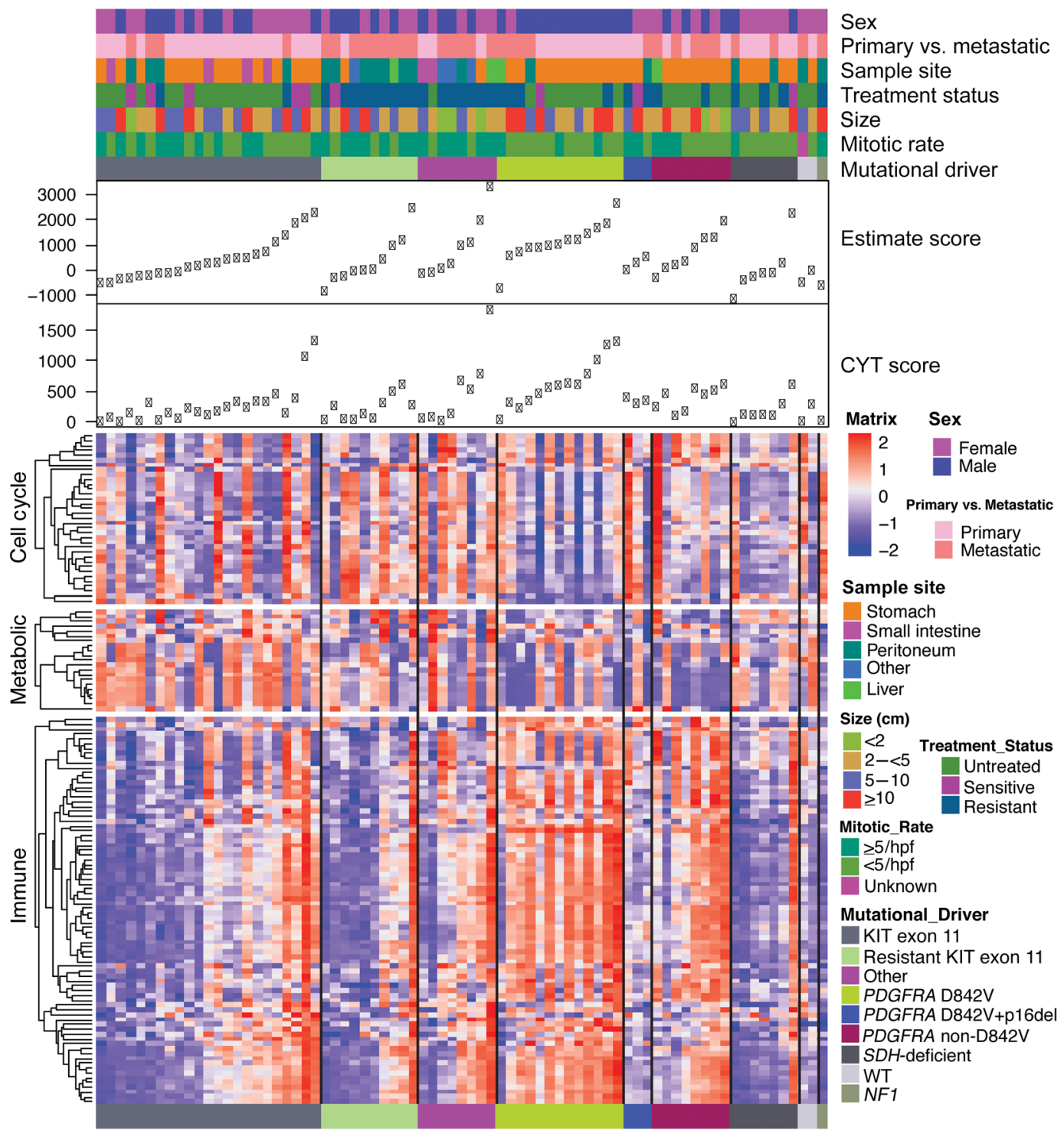

Figure 1. ssGSEA identifies immune cell pathway enrichment in PDGFRA-mutant GIST. sSGSEA of 75 GIST specimens, organized by mutational driver and increasing ESTIMATE score. Unsupervised row clustering grouped gene sets into 3 major categories based on cell cycle pathways, metabolic pathways, and immune pathways. Clinicopathologic characteristics of the 75 GIST specimens are shown in the annotation and in Supplemental Table 1.

scape. Finally, we identified the top immune features correlating with high PD-1 and PD-L1 expression across all GIST specimens to identify potential barriers to and synergistic opportunities for successful immune checkpoint blockade in GIST.

\section{Results}

Single-sample gene set enrichment analysis identifies immune cell pathway enrichment in PDGFRA-mutant GIST. We performed RNA-Seq and principal component analysis (PCA) of 75 human GIST specimens comprising various mutation types $(n=37$ KIT-mutant, $n=24$ PDGFRA-mutant, $n=7 S D H$-deficient, $n=4$ multiple drivers, $n=2 \mathrm{WT}$, and $n=1$ NF1-mutant; Supplemental Table 1; supplemental material available online with this article; https://doi.org/10.1172/JCI124108DS1). The combined PCA of all mutational drivers demonstrated clustering by mutational subtype (Supplemental Figure 1, top), which was most apparent in
PDGFRA-mutant and SDH-deficient GISTs (Supplemental Figure 1, bottom). Notably, KIT-mutant GISTs appeared to cluster into 3 distinct groups, which upon closer inspection of clinicopathologic features was related to treatment status and tumor location.

Given what our group has discovered regarding the immune infiltrate of GIST $(13-15,17)$, the metabolic characteristics of imatinib-treated GIST (18), and the association of cell cycle activity with GIST aggressiveness $(19,20)$, we used our sequencing data to perform single-sample gene set enrichment analyses (ssGSEAs) focused on immune, metabolic, and cell cycle pathways (Figure 1). Using published gene sets (21), along with assessment of previously published metabolic, cell cycle, and immune pathways (22), we identified 136 gene sets for inclusion in ssGSEA (Supplemental Table 2).

ESTIMATE and CYT scoring, which infer the quantity and cytolytic activity of the immune infiltrates, respectively (23, 24), revealed that KIT-mutant GIST had a range of immune cell 
infiltration and cytolytic activity (Figure 1). Meanwhile, $S D H$ deficient, NF1-mutant, D842V-mutant GISTs with a concurrent CDKN2A (p16) deletion, and WT GISTs (defined as non-KIT, non-PDGFRA, non-RAS-activated, and non-SDH-deficient, as previously described; ref. 25) exhibited generally low ESTIMATE and CYT scores and a lack of immune cell pathway enrichment.

Conversely, ESTIMATE, CYT, and ssGSEA identified increased immune cell infiltration, greater immune cell activity, and a significant enrichment of immune-related gene sets in PDGFRA-mutant GISTs - an unexpected finding that suggested that PDGFRA-mutant GISTs were more immunogenic than other GIST mutations (Figure 1). Cell cycle activity pathways did not clearly correlate with immune cell infiltration across all GIST driver mutation types, while metabolic activity appeared to inversely correlate with the quantity of immune infiltrate, specifically in KIT exon 11-mutant GISTs. Overall, these data show that while tumors driven by a particular GIST mutation can have variable immune profiles, PDGFRA-mutant GIST contains the strongest gene expression-based immune signature when compared with other GIST mutations.

PDGFRA-mutant GIST is more immunologically active than KIT-mutant GIST. In order to validate our ssGSEA finding that PDGFRA-mutant GIST was highly enriched in immune cell quantity and cytolytic activity, we first compared the clinicopathologic characteristics of all KIT- and PDGFRA-mutant GISTs (Supplemental Table 3; $n=61$ ). Though not associated with immune cell infiltration in GIST, tumor size, mitotic rate, and tumor location are predictive of GIST aggressiveness and recurrence-free survival after surgical resection (26). PDGFRA-mutant GISTs were more commonly found in stomach when compared with KIT-mutant GISTs, and there were significantly more males in our PDGFRA-mutant GIST cohort. There was also a trend toward decreased mitotic activity in PDGFRA-mutant when compared with KIT-mutant GISTs (Supplemental Table 3). ESTIMATE and CYT scores, however, did not correlate with mitotic rate across all KIT- and PDGFRA-mutant GISTs (Supplemental Figure 2A). CYT score and the overall number of CD45 mRNA transcripts were significantly higher in PDGFRA-mutant when compared with KIT-mutant GISTs (Figure 2A), while there was also a trend toward increased ESTIMATE score and CD8 mRNA expression, suggesting that PDGFRA-mutant GISTs may exhibit greater immune cell infiltration and activation than KIT-mutant GISTs.

Since we observed significant differences in tumor location between KIT- and PDGFRA-mutant GISTs, and metastatic lesions and treatment status have also been shown to alter the immune infiltrate in GIST $(14,27)$, we then compared only UPG PDGFRAand KIT-mutant GISTs to minimize confounders and maximize the potential to observe important biologic differences that may be related to the oncogenic driver (Supplemental Table $4 ; n=22$ ). UPG PDGFRA- and KIT-mutant GISTs with a low mitotic rate exhibited significantly higher ESTIMATE scores when compared with UPG GISTs with a high mitotic rate (Supplemental Figure $2 \mathrm{~B})$, suggesting that immune cell infiltration may be partly related to GIST aggressiveness when controlling for tumor location and treatment status. However, when controlling for the mutational driver in addition to tumor location and treatment status, mitotic rate did not inversely correlate with immune cell infiltration
(Supplemental Figure 2C), implying that the oncogenic driver may also be contributing to differences in immune response. In fact, ESTIMATE score, CYT score, and the mRNA expression of CD45 were significantly greater in UPG PDGFRA-mutant GISTs when compared with UPG KIT-mutant GISTs, and expression of CD8 mRNA was also increased (Figure 2B). Furthermore, gene set enrichment analyses (GSEAs) showed that the overall immune response pathway, adaptive immune response pathway, antigen binding pathway, and $\alpha / \beta$ T cell activation pathway (Figure $2 \mathrm{C}$ ), as well as lymphocyte activation, lymphocyte differentiation, and B cell activity pathways (data not shown), were more significantly enriched in UPG PDGFRA-mutant compared with KIT-mutant GISTs, suggesting that the difference in immune infiltration and activity may be related to the oncogenic driver.

To further validate the observed differences in immune cell infiltrate between PDGFRA- and KIT-mutant GISTs, we performed IHC staining for CD45 and CD8 on KIT- and PDGFRA-mutant GISTs, including additional tumors not included in the sequencing cohort (Figure 2D). On gross microscopic examination, it appeared that PDGFRA-mutant GISTs contained more $\mathrm{CD} 45^{+}$and $\mathrm{CD} 8^{+}$cells, with a proportion of immune cells clustered around perivascular structures, a finding that we have found to be associated with adaptive immunity (our unpublished observations). Quantification of CD45 and CD8 IHC staining, including staining of the most infiltrated and cytolytically active KIT-mutant GIST in the KIT-mutant cohort (human GIST 203), showed that PDGFRA-mutant GISTs had significantly more $\mathrm{CD} 45^{+}$and $\mathrm{CD} 8^{+}$ cells per $\times 20$ high-power field $(\mathrm{HPF})$ than KIT-mutant GISTs (Figure 2E). Moreover, flow cytometric analysis of KIT- and PDGFRA-mutant GIST specimens confirmed that PDGFRA-mutant GISTs harbored significantly more CD $45^{+}$immune cells than KIT-mutant GISTs (Figure 2E). Together, these findings confirm that in PDGFRA-mutant GISTs, immune cells are more numerous and have higher cytolytic activity than in KIT-mutant GISTs with similar clinicopathologic features.

CIBERSORT and differential gene expression analysis identify unique immune signatures in GIST. To discover additional differences in the immune landscape between UPG PDGFRA- and UPG KIT-mutant GISTs, we profiled a previously defined set of 117 relevant immune features (Supplemental Table 5) $(28,29)$. In addition to ESTIMATE and CYT scores, this profile included scores derived from CIBERSORT, which infers various immune cell frequencies through RNA-Seq deconvolution, as well as 93 relevant immune-related genes. UPG PDGFRA-mutant GISTs appeared to have higher proportions of intratumoral $\mathrm{CD} 4^{+} \mathrm{T}$ cell and macrophage expression (Figure 3, middle), while UPG KIT-mutant GISTs primarily expressed CD4 and macrophage signatures only in the most immune cell-infiltrated tumors. Furthermore, B cell and $\mathrm{CD}^{+} \mathrm{T}$ cells signatures were also expressed in GISTs with high ESTIMATE and CYT scores, confirming what we and others have shown regarding the immune cell prevalence in GIST $(14,27)$.

Differential gene expression (DGE) analysis revealed that 20 of $93(21.5 \%)$ immune-related genes were significantly differentially regulated between UPG KIT- and UPG PDGFRA-mutant GISTs (bold indicates $P<0.05$; Figure 3, bottom). Specifically, UPG PDGFRA-mutant GISTs expressed significantly more CCR5, BTLA, CD96, CD48, TNFRSF9, TNFSF8, CCR4, CXCL11, 
A
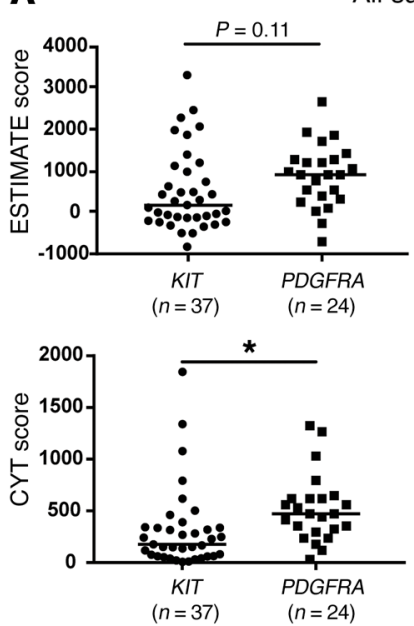

C IMMUNE_RESPONSE

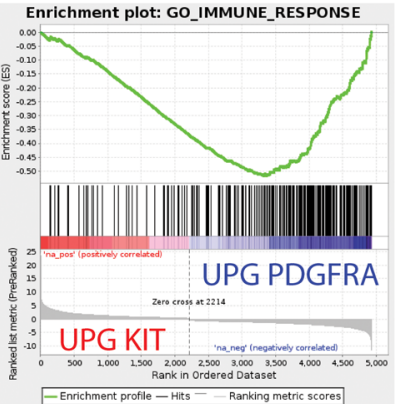

$\mathrm{NES}=-2.79 ; \mathrm{FDR}<0.001$
All samples
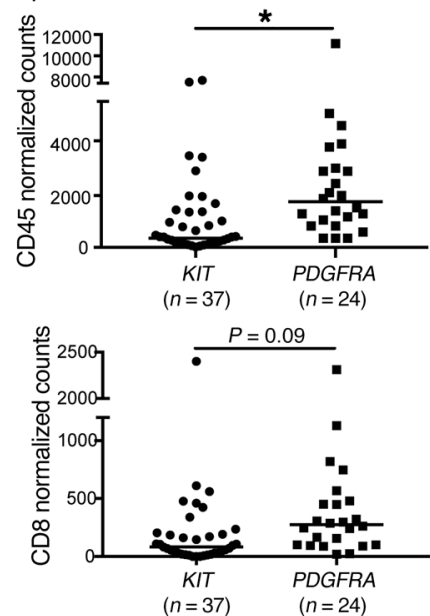

ADAPTIVE_IMMUNE_RESPONSE

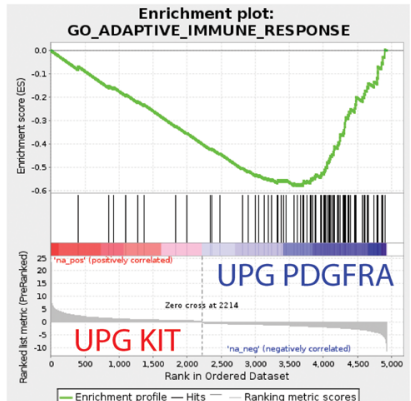

$\mathrm{NES}=-2.58 ; \mathrm{FDR}<0.00$
B
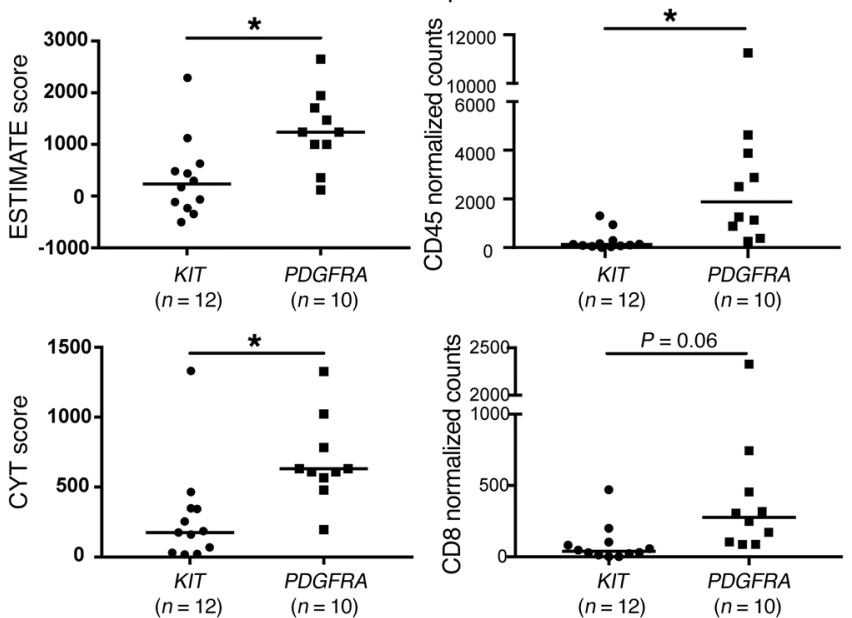

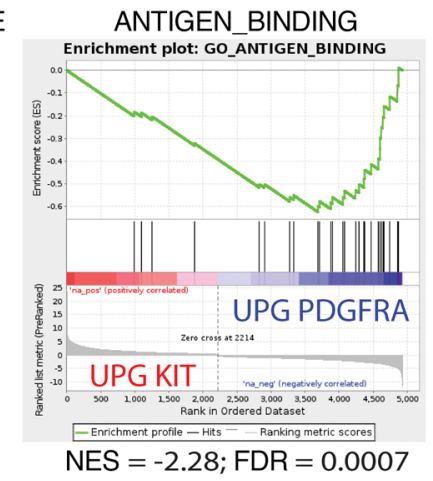

ALPHA_BETA_T_CELL_ACTIVATION Enrichment plot:
Go_ALPHA_BETA_T_CELL_ACTIVATION

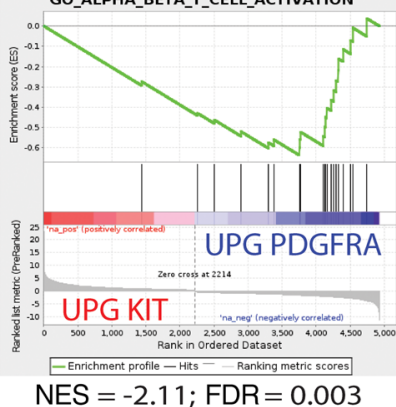

E
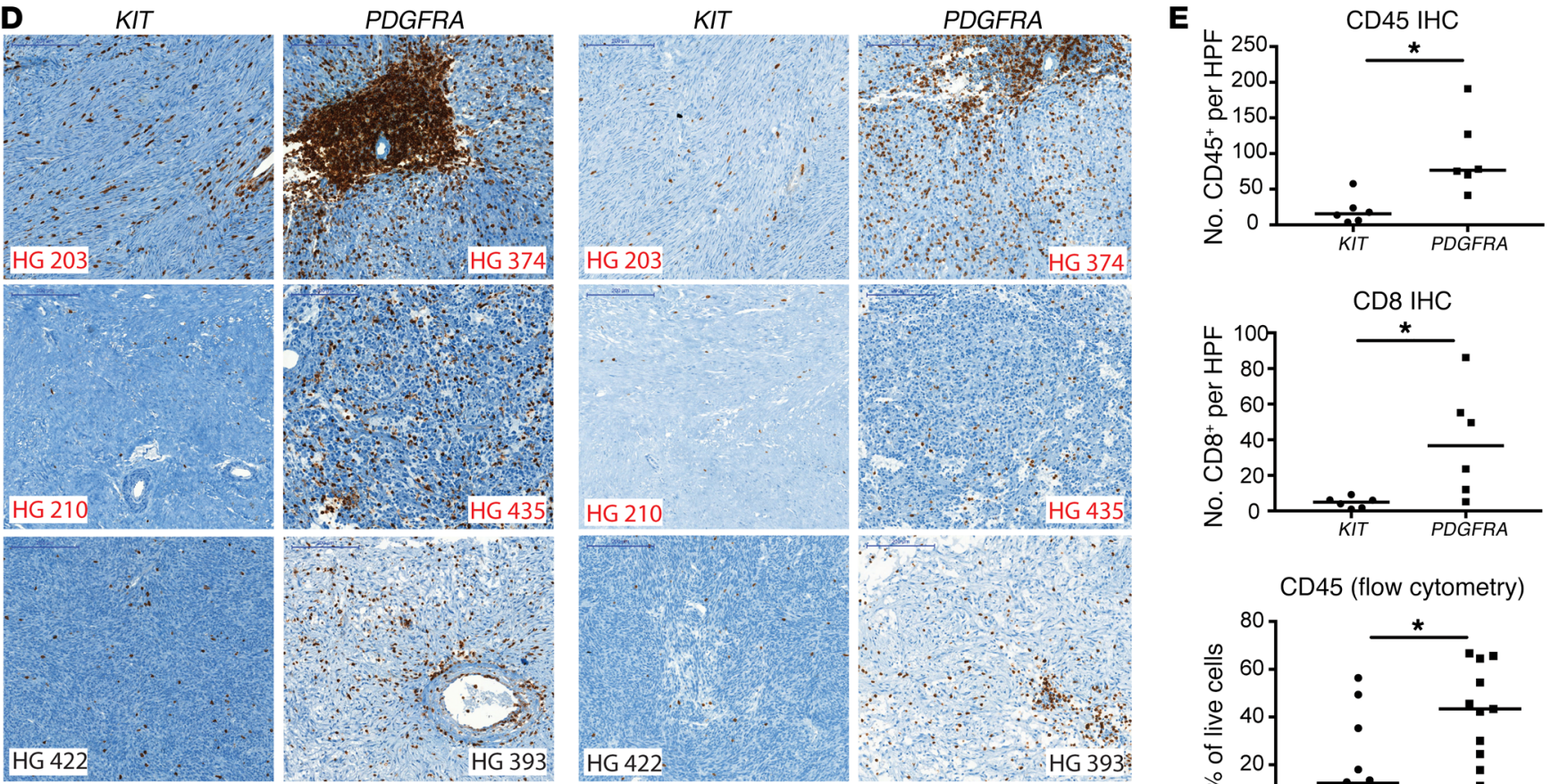

CD8

CD45

PDGFRA

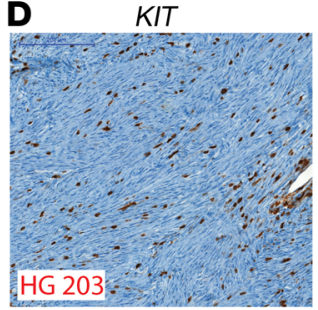


Figure 2. PDCFRA-mutant GIST is more immunologically active compared with KIT-mutant GIST. (A) ESTIMATE and CYT scores (left) and CD45 and CD8 normalized counts (right) of all KIT- and PDGFRA-mutant GISTs ( $n=61$; Supplemental Table 3). (B). ESTIMATE and CYT scores (left) and CD45 and CD8 normalized counts (right) of UPG KIT- and PDGFRA-mutant GISTs ( $n=22$; Supplemental Table 4). (C) GSEA showing multiple immune pathways enriched in UPG PDGFRA-mutant compared with UPG KIT-mutant GISTs. NES, normalized enrichment score. (D) $\times 10$ magnification CD45 and CD8 IHC staining in KIT- and PDGFRA-mutant GISTs. Red text indicates specimen was included in RNA-Seq cohort, while samples represented in black text were not included. Representative samples of $n=6$ per group are shown. (E) CD45 (top) and CD8 (middle) quantification of IHC staining. $n=6$ per group. The number of CD45 $5^{+}$ and $\mathrm{CD} 8^{+}$cells per HPF was calculated by examining $5 \mathrm{HPFs}$ per tumor and plotting the average per tumor. Bottom: CD45 expression by flow cytometry (for specimens in which flow cytometry data were available). ${ }^{*} P<0.05, t$ test. Bars indicate median.

CXCR4, KDR, IL6R, TNFRSF8, TNFSF14, TIGIT, TNFRSF17, HLA-DQA2, CXCL14, and CXCL12. Meanwhile, KIT-mutant GISTs expressed significantly more TNFSF18 and MICA. Together, these data suggest that PDGFRA- and KIT-mutant GISTs elicit a different quality of immune infiltrate, which may ultimately provide driver-specific strategies for immunotherapy.

PDGFRA- and KIT-mutant GISTs have distinct signaling and cytokine signatures. To determine whether intracellular signaling or cytokine differences could be contributing to the observed differences in immune infiltration between UPG PDGFRA-mutant and UPG KIT-mutant GISTs, we utilized GSEA to detect differences in these pathways. On GSEA, we first observed enrichment of a known PDGFRA-mutant GIST signaling pathway gene set from a prior report analyzing 3 PDGFRA-mutant GISTs (Figure 4A, top) (30). Compared with UPG KIT-mutant GISTs, UPG PDGFRAmutant GISTs also demonstrated significant transcriptional activation of the PI3K signaling pathway (Figure 4A, bottom), which may have been driven by expression changes in PDGFRA, RELN, $K D R$, or $E R K$, 4 genes that have been found to be significantly differentially expressed between PDGFRA- and KIT-mutant GISTs not only in our cohort but also previously (30). Furthermore, many cytokine and chemokine pathways were significantly upregulated in UPG PDGFRA-mutant GISTs (Figure 4B), which we found to be related to increased expression of CXCL14, CCL7, CCL19, VEGFA, and KITLG (Figure 4C).

The differences observed in CXCL14 expression were particularly intriguing. First, CXCL14 mRNA was expressed at significantly higher levels in nearly all UPG PDGFRA-mutant GISTs. Quantitative real-time PCR (qRT-PCR) of bulk human PDGFRA- and KIT-mutant tumors also revealed that PDGFRA-mutant GISTs produced approximately 10 times more CXCL14 mRNA than KIT-mutant tumors, and 21 times more CXCL14 mRNA than the KIT-mutant GIST-T1 (Figure 4D, left) and GIST-882 cell lines (data not shown). Finally, CXCL14 has been shown to contribute to $\mathrm{NK}^{+}, \mathrm{CD}^{+}, \mathrm{CD}^{+}$, and regulatory $\mathrm{T}$ cell chemotaxis and tumor regression in other tumor models $(31,32)$. UPG PDGFRA-mutant GISTs expressed CXCL14 mRNA at levels higher than those shown to lead to immune-mediated tumor regression in $\mathrm{HPV}^{+}$head, neck, and cervical cancers, while KIT-mutant tumors expressed CXCL14 mRNA at levels below this threshold (Figure 4D, right). Together, these findings suggest that CXCL14 expression may contribute to the observed differences in immune infiltration between $P D G$ FRA- and KIT-mutant GISTs $(31,32)$.

PDGFRA mutation produces multiple HLA-diverse, strong binding neoepitopes. Given the association of neoantigen presentation with inflamed tumor/immune microenvironments in other malignancies, we hypothesized that PDGFRA-mutant GISTs may produce more potent neoepitopes when compared with KIT-mutant GISTs. To explore this, we first mapped out the 8-, 9-, and 10-mer neoepitopes produced by all driver mutations in our cohort, generating neoepitopes with the mutation placed at each amino acid position in the mutant peptide. Then, we tested the binding affinity of each neoepitope to the matched, patient-specific HLA type in which that mutation was found.

First, we wanted to determine whether total neoepitope burden and the number of high-affinity neoepitopes produced by each GIST specimen correlated with immune infiltration or cytolytic activity in GIST, since neoepitope burden has been shown to correlate with increased response to immunotherapy in a variety of cancers $(33,34)$. However, total neoepitope burden and the number of high-affinity ( $<500 \mathrm{nM}$ binding) or very high-affinity $(<50 \mathrm{nM}$ binding) neoepitopes did not correlate with immune infiltration or cytolytic activity across all mutations in the GIST cohort (Figure 5A) or across KIT- and PDGFRA-mutant GISTs specifically (data not shown).

We observed that the majority of patients with an oncogenic mutation in PDGFRA or KIT produced at least one high-affinity neoepitope ( $<500 \mathrm{nM}$ binding), regardless of mutation subtype (Figure 5B). We also found that the $D 842 \mathrm{~V}$ mutation produced 34 unique, high-affinity neoepitope:HLA-specific binding combinations across the predicted HLA types in our cohort of 16 D842V-mutant patients (Figure 5B), while KIT exon 11 and other PDGFRA mutations on average produced only 2-3 per mutation (Supplemental Table 6). The $D 842 \mathrm{~V}$ mutation generated 6 different neoepitopes that bound to 12 different HLA types in 14 patients in our cohort (Supplemental Table 7), one of which is the most common HLA type in the United States and present in $95.7 \%$ of White individuals $\left(\mathrm{HLA}^{\star} \mathrm{AO} 2 \mathrm{O}: 01\right)(35,36)$, suggesting that this mutation could produce an immune response in a wide variety of patients, whereas KIT exon 11 neoepitopes bound to less-prevalent HLA types.

Since the number of patients with $D 842 \mathrm{~V}$ mutations $(n=16)$ exceeded the number of patients with any individual KIT mutation ( $n=1-4$, Supplemental Table 7), and this may obscure our HLA observations, we explored mutation-specific neoepitope binding diversity more broadly against all HLA types validated by NetMHCPan3.0 (Figure 5C). Again, nearly all PDGFRA and KIT GIST mutations produced at least one high-affinity neoepitope capable of binding to many different HLA types. D842V neoepitopes bound with very high affinity ( $<50 \mathrm{nM}$ binding) to $\mathrm{HLA}^{\star} \mathrm{A02}$ :01 and many other prevalent HLA types. Interestingly, 50\% (4 of 8) of all PDGFRA mutations produced at least one very high-affinity neoepitope ( $<50 \mathrm{nM}$ binding) to $\mathrm{HLA}^{\star} \mathrm{A02}$ :01, compared with only $14 \%$ of KIT mutations ( 2 of 14 ). Thus, while all KIT- and PDGFRA-mutant GISTs appear to produce highaffinity neoepitopes, PDGFRA-mutant GISTs appear to produce more high-affinity neoepitopes to more common HLA types. 


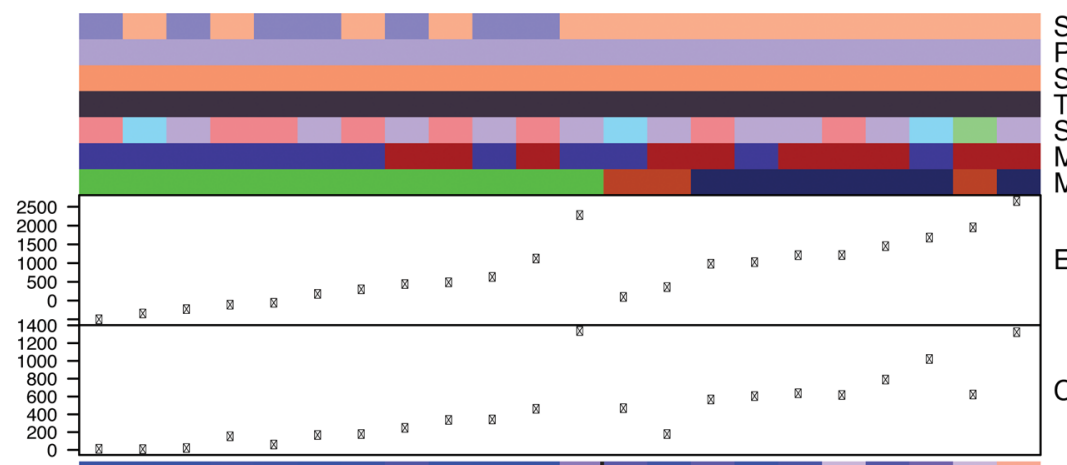

Sex

Primary vs. metastatic

Sample site

Treatment status

Size

Mitotic rate

Mutational driver

Estimate score

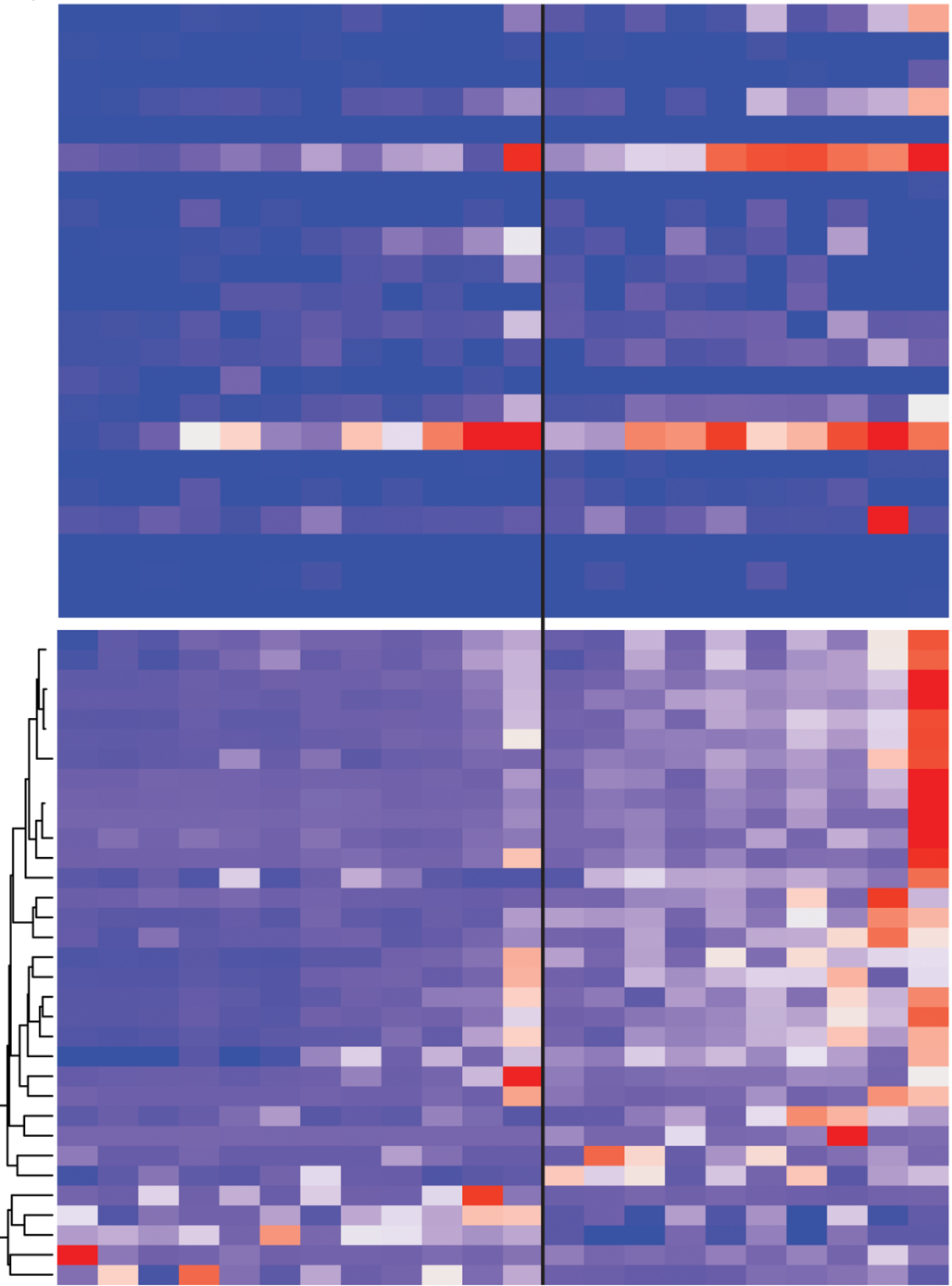

B cells naive

$B$ cells memory

Plasma cells

T cells CD8 ${ }^{+}$

$T$ cells $\mathrm{CD}^{+}{ }^{+}$naive

T cells $\mathrm{CD} 4^{+}$memory resting

$T$ cells $\mathrm{CD} 4^{+}$memory activated

$T$ cells follicular helper

Tregs

T cells $\gamma \delta$

NK cells resting

NK cells activated

Monocytes

Macrophages M0

Macrophages M1

Macrophages M2

Dendritic cells resting

Dendritic cells activated

Mast cells resting

Mast cells activated

Eosinophils

Neutrophils

CD86

HAVCR

CCR5

BTLA

CD96

CD48

TNFSF8

CCR4

CXCL11

CXCR4

CXCL10

ULBP

KDR

IL6R

TNFSF 14

CD160

$P D C D$

TIGIT

$C D 244$
$B 2 M$

BDM

TNFRSF17

HLA-DQA2

CXCL14

VTCN1

TGFBR1

TNFSF18

MICA

CXCL12
TMEM173

Sex
Female
Male
Sample site
Gastric
Primary vs. metastatic
Primary
Treatment status
Untreated
Size (cm)
$>10$
$5-10$
$2-<5$
$<2$
Mitotic rate
$>5 / h p f$
$<5 / h p f$
Mutational driver
KIT exon 11
PDGFRA non-D842V
PDGFRA D842V

Figure 3. CIBERSORT and DGE analysis identify unique immune signatures in GIST. CIBERSORT (middle) and immune gene expression (bottom) of UPG KIT- and UPG PDGFRA-mutant GISTs, organized by mutational driver and increasing ESTIMATE score $(n=22)$. Unsupervised row-normalized clustering of genes shows grouping of genes into distinct groups, suggestive of oncogene-driven immune profiles. Genes shown in blue were significantly enriched in UPG PDCFRA-compared with UPG KIT-mutant, while genes in green were significantly enriched in UPG KIT-compared with UPG PDGFRA-mutant samples. Enrichment was considered as an adjusted $P<0.1$ as calculated by DESeq2 for $R$, while boldface for gene names indicates a significant difference, with an adjusted $P<0.05$. Clinicopathologic characteristics of the UPG GIST specimens are shown in the annotation and in Supplemental Table 4.

Given that GIST is often driven by a single oncogenic mutation, and GIST harbors a rich immune infiltrate, this raises the possibility that oncogenic GIST mutations produce neoepitopes that may classify as what has recently been provocatively described as high-quality neoepitopes (37).
Machine learning identifies immune gene signatures for GIST. Through bioinformatics prediction and biologic validation methods, it appears that in PDGFRA-mutant GIST, immune cells are more numerous and have higher cytolytic activity than in KITmutant GISTs, and both subsets contain a unique immune pro- 
A

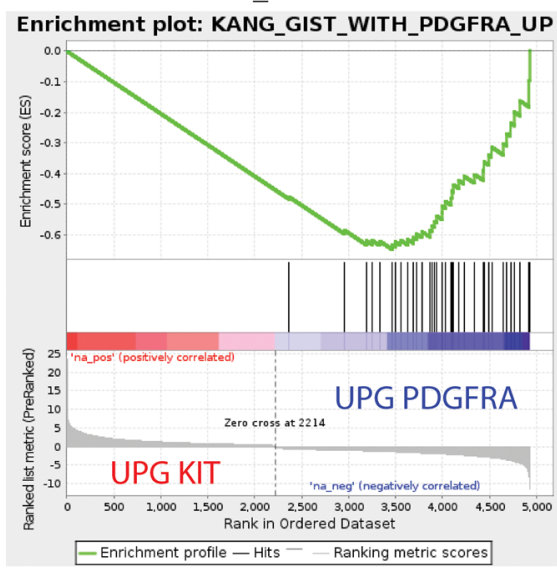

$\mathrm{NES}=-2.49 ; \mathrm{FDR}<0.001$

PI3K SIGNALING

Enrichment plot:

GO_POSITIVE_REGULATION_OF_PHOSPHATIDYLIN OSITOL_3_KINASE_SIGNALING

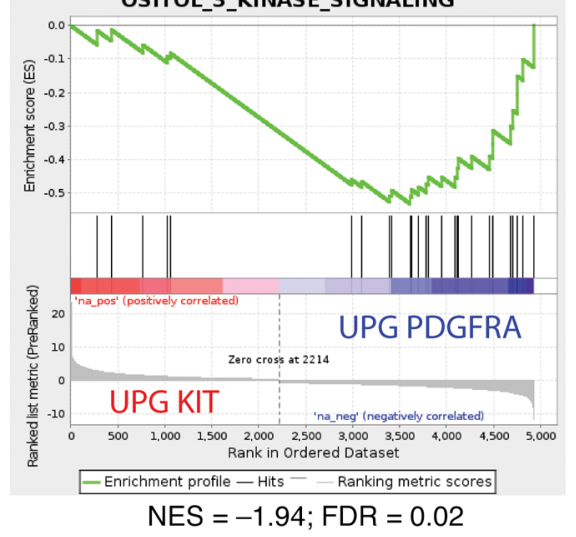

C

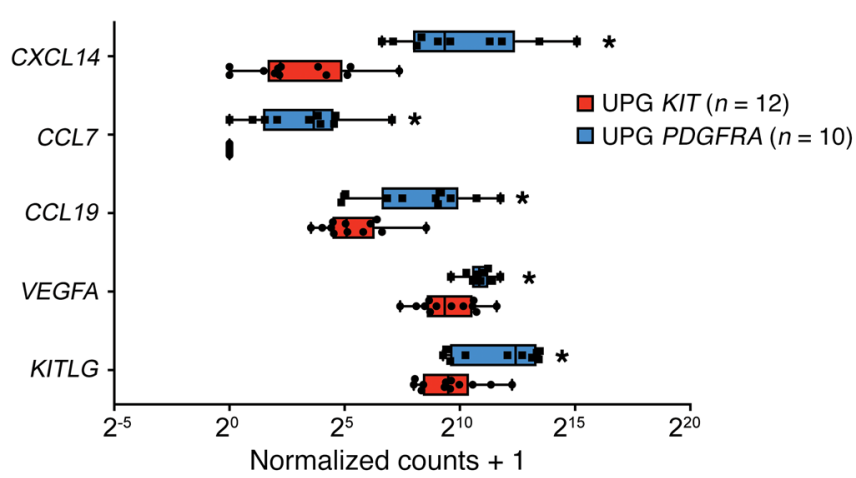

Cytokine expression (RNA-Seq)
B CHEMOKINE_SIGNALING Enrichment plot:
GO_CHEMOKINE_MEDIATED_SIGNALING_PATHWA

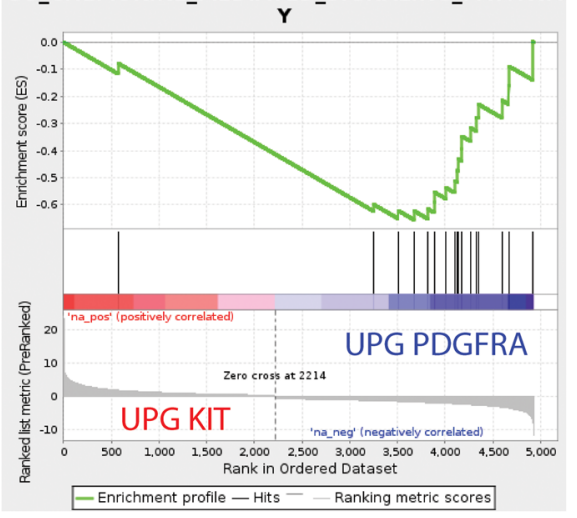

$\mathrm{NES}=-2.16 ; \mathrm{FDR}<0.002$

KEGG_CYTOKINE Enrichment plot:
KEGG_CYTOKINE_CYTOKINE_RECEPTOR_INTERACT ION

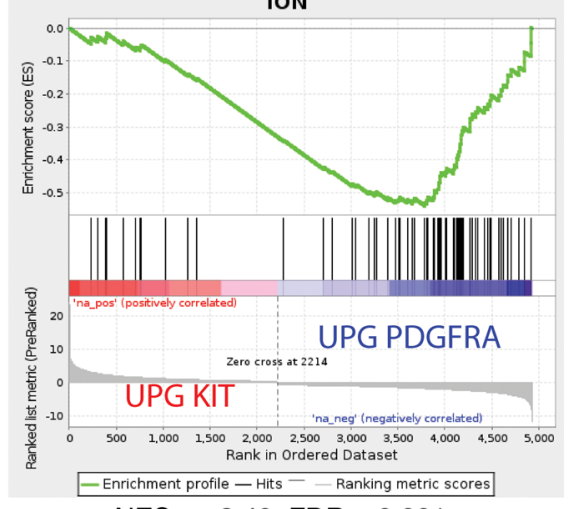

$\mathrm{NES}=-2.40 ;$ FDR $<0.001$
CYTOKINE RECEPTOR ACTIVITY Enrichment plot: GO_CYTOKINE_RECEPTOR_ACTIVITY

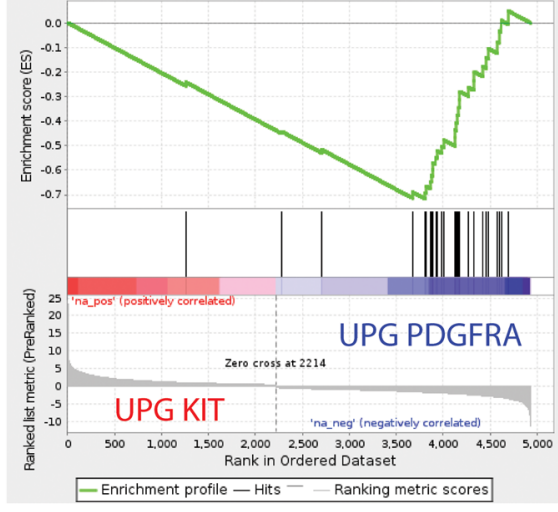

$\mathrm{NES}=-2.61 ;$ FDR $<0.001$

CELL_CHEMOTAXIS Enrichment plot: GO_CELL_CHEMOTAXIS

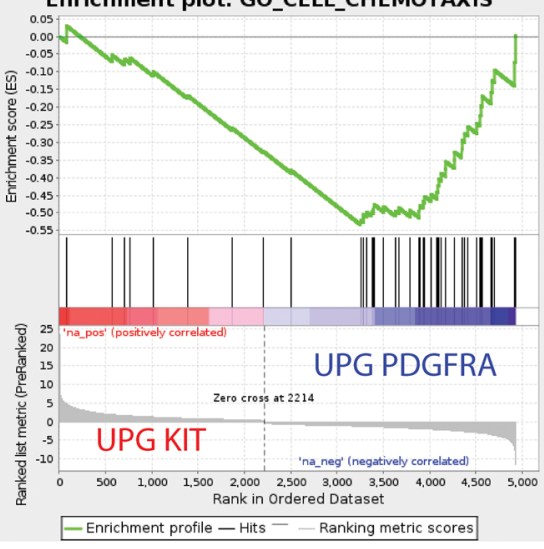

$\mathrm{NES}=-2.10 ; \mathrm{FDR}=0.004$
D

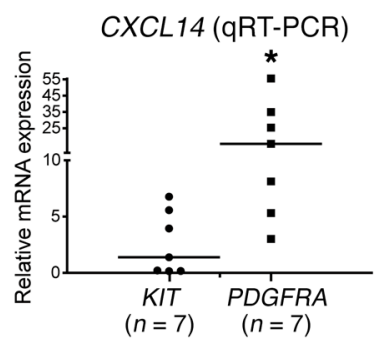

CXCL14

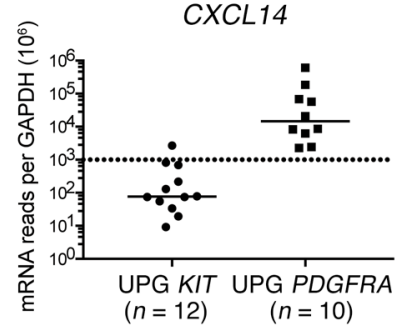

Figure 4. PDGFRA- and KIT-mutant GISTs have distinct signaling and cytokine signatures. GSEA showing enrichment of (A) PDCFRA signaling, PI3K signaling, and (B) cytokine signaling pathways in UPG PDGFRA- compared with UPG KIT-mutant GISTs $(n=22)$. (C) Distribution of cytokines between UPG KIT- and UPG PDGFRA-mutant GISTs, by RNA-Seq. Adjusted $P<0.05$ as calculated by DESeq2. All data points are shown; boxes define the interquartile range, with whiskers extending to lowest and highest data points. (D) Left: Relative CXCL14 mRNA expression by qRT-PCR in KIT- $(n=7)$ and PDGFRAmutant $(n=7)$ GISTs from the RNA-Seq cohort, compared with the GIST-T1 cell line (expression set at 1; data not shown). Right: CXCL14 mRNA expression relative to GAPDH $\times 10^{6}$ in UPG KIT- and PDGFRA-mutant GISTs. Horizontal dotted line represents CXCL14 mRNA expression needed to induce tumor regression (31). ${ }^{*} P<0.05, t$ test. Bars indicate the median.

file. This makes characterizing a global immune infiltrate in GIST challenging and suggests that specific immunotherapy approaches may ultimately need to be targeted to the specific oncogenic mutation in GIST. We therefore sought to define the most import- ant immune features differentiating PDGFRA- and KIT-mutant GISTs through machine learning, which is an unbiased approach not dependent on previously defined gene set analysis, allowing for novel and impartial observations. We specifically used all KIT- 
A
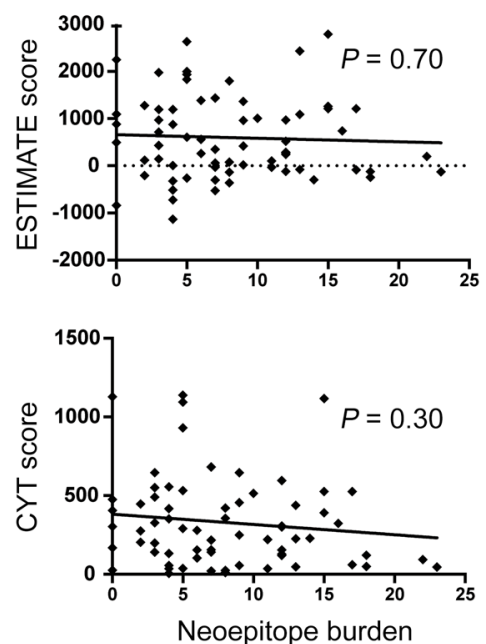
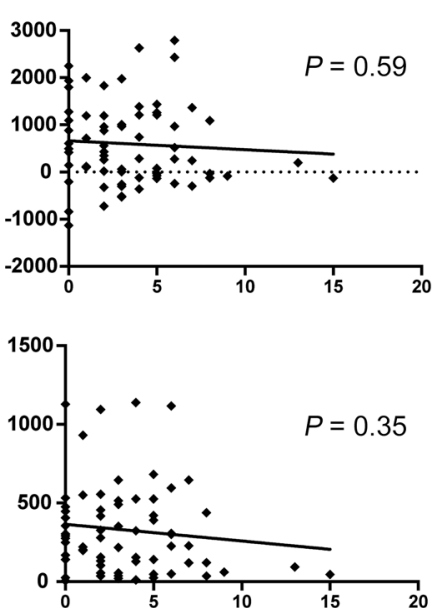

No. of neoepitopes $<500 \mathrm{nM}$ binding
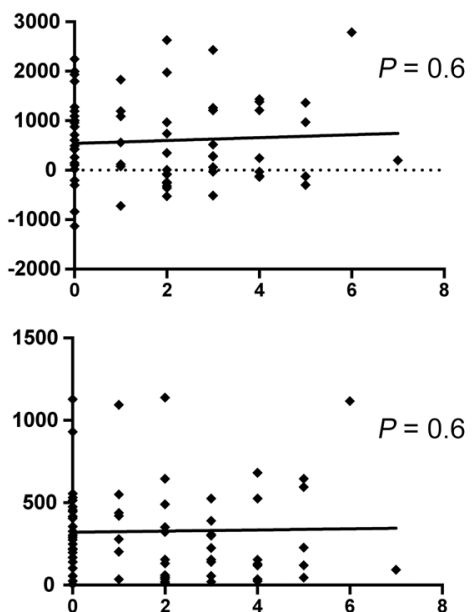

No. of neoepitopes $<150 \mathrm{nM}$ binding

B

Patients with predicted high-affinity neoepitopes
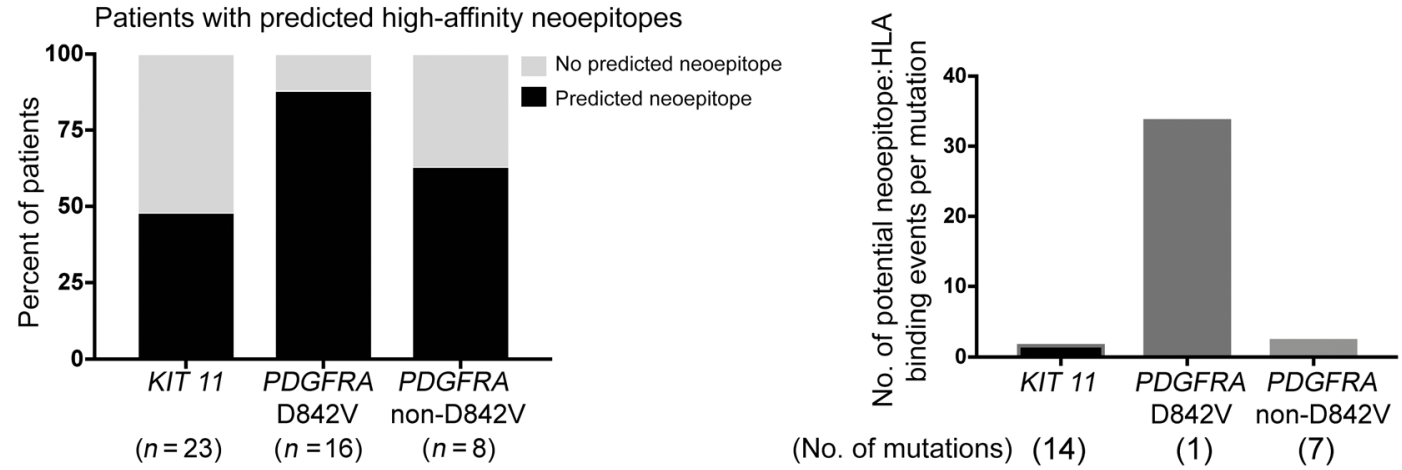

(No. of mutations) (14)

(1)

(7)

C

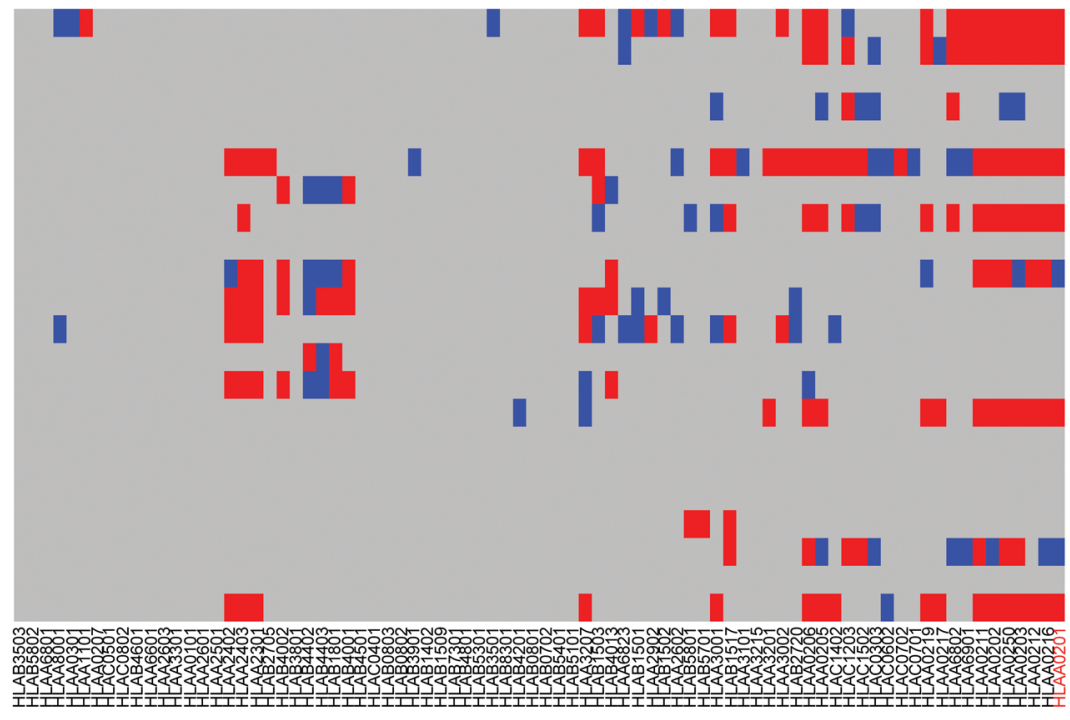

PDGFRA D842V PDGFRA_D8421 PDGFRA V561D PDGFRA_1843_D846del PDGFRA D842 D846>E PDGFRA S566 E571delinsR PDGFRA_L595_G596ins PDGFRA 1843_D846deIMHD KIT11 V559D

KIT11 W557 K558del KIT11_F584_P585ins KIT11 P551del KIT11_1563_L576del KIT11_K558_V559del KIT11 K558 V559delinsN KIT11_W557_V560>P KIT11_W557R KIT11 V560D KIT11_577_578ins KIT11_K550_K558del KIT11_V559_E561del KIT11 E554 V559>V

Figure 5. PDCFRA mutation produces multiple HLA-diverse, strong binding neoepitopes. (A) Pearson's correlation of ESTIMATE (top) and CYT (bottom) scores with total neoepitope burden (left), number of high-affinity neoepitopes (middle), and number of very high-affinity neoepitopes (right) among all GIST samples $(n=75)$. (B) Left: Percentage of patients with the indicated mutation whose mutation produced a predicted high-affinity neoepitope. Right: Number of potential neoepitope:HLA binding events produced by mutation type, averaged over the number of mutations per group. (C) Heatmap of the binding affinities of KIT and PDGFRA mutation-specific neoepitopes to all validated NetMHCPan 3.0 HLA types. Clinicopathologic characteristics of the GIST specimens are shown in Supplemental Table 3. Additional details regarding mutations, neoepitopes, and HLA types used to create this heatmap are shown in Supplemental Tables 6 and 7. 
and PDGFRA-mutant GISTs (Supplemental Table 3; $n=61$ ), as well as only UPG KIT- and PDGFRA-mutant GISTs (Supplemental Table $4 ; n=22$ ) to develop 2 random forest models, and assumed that these immune differences would most likely be related to the oncogenic driver.

The combination of salient immune features capable of most accurately profiling a PDGFRA- or KIT-mutant GIST were identified based on each feature's "importance," which is calculated as part of caret for R's implementation of randomForest (38). The feature importance metric is a measure indicating how useful a feature is for separating samples into their distinct classes. The features with the highest importance provide the classifier with the highest increase in performance.

We included all 117 immune features to initially create the model (Supplemental Table 5) and subsequently retrained the model using fewer features. We noted that including more features in the model increased the model's performance on the training set, but decreased the model's performance on the test set, a phenomenon known as overfitting (Supplemental Figure 3A). Therefore, to prevent overfitting, we retrained the model using only the 6 most important features, which we believe will broaden the applicability of our model to future GIST samples. When we restricted the model to the 6 most relevant features (Figure 6A), 27 of $30 \mathrm{KIT}$-mutant GISTs were correctly classified as KIT-mutant in the training set, while 14 of 20 PDGFRA-mutant GISTs were correctly assigned as PDGFRA-mutant (sensitivity: 70\%, specificity: 90\%). The top immune features included CXCL14, TGFBR1, TNFSF9, MICA, TNFRSF25, and CD96 (Figure 6B). Notably, the PDGFRA-mutant tumors that were incorrectly classified as KIT-mutant by our model had lower ESTIMATE and CYT scores than correctly classified PDGFRA-mutant tumors (Supplemental Figure $4 \mathrm{~A}$ ), while $K I T$-mutant tumors incorrectly classified as PDGFRA-mutant had more variable CXCL14 mRNA expression and higher TGFBR1 and CD96 mRNA expression than correctly classified KIT-mutant tumors (Supplemental Figure 4B). In our separate cohort of testing samples $(n=11)$, the 6 -feature model correctly identified KIT- and PDGFRA-mutant GISTs with $91 \%$ accuracy (Figure 6C). To demonstrate the importance of these 6 immune features in classifying KIT- and PDGFRA-mutant GISTs, we excluded these features and retrained our random forest model, which reduced classifier performance and model accuracy from $91 \%$ to $72.7 \%$ (Supplemental Figure 3B).

We further validated our model on an external cohort of GISTs from the Complexity Index in Sarcomas (CINSARC) study $(n=12$; ref. 39). Application of our immune profiling model to the CINSARC cohort correctly differentiated KIT- and PDGFRA-mutant GISTS with $83.3 \%$ accuracy (Figure 6C). Similar results were obtained when we created a model using only UPG PDGFRAand KIT-mutant GISTs (Figure 6, D-F), in which CXCL14, IDO, TNFSF14, KDR, MICA, and TNFRSF9 were the most important features defining the model.

We employed the same machine learning approach to identify gene expression-based immune features associated with high PD-1 and PD-L1 expression across all GISTs $(n=75)$ in order to discover novel therapeutic targets and potential barriers to immune checkpoint blockade in GIST. Interestingly, our 6-feature model showed that increased CD27 expression was highly related to
PD-1 upregulation (sensitivity: 90\%, specificity: 87.1\%, Figure 7A), along with PDCD1LG2, BTLA, CD40, CXCL14, and MICB, suggesting that these pathways may represent synergistic opportunities for PD-1 immunomodulation in GIST (Figure 7B). Using only these 6 features, our model was able to correctly predict high PD-1 expression in $92.9 \%$ of the testing cohort and $66.7 \%$ of the external CINSARC GIST cohort (Figure 7C).

PD-L1 mRNA expression correlated with PD-L1 protein expression in our cohort (Supplemental Figure 5; see complete unedited gel in the supplemental material). When training our PD-L1 random forest model, expression of key antigen-presenting machinery, including B2M, HLA.DRA, and TAP2, and the immune-responsive cytokines CXCL10 and LTA were predictive of increased PD-L1 expression across all GISTs (sensitivity: 76.7\%, specificity: $87.1 \%$, Figure 7D), suggesting a relationship between immune checkpoint ligand upregulation and immune recognition (Figure 7E). Applying the model of these antigen-presenting machinery features and immune cytokines, along with PD-L2, which was also identified as a feature predictive of high PD-L1 expression, our model correctly identified high PD-L1 expression with $85.7 \%$ accuracy in the testing cohort and $91.7 \%$ in the CINSARC GIST cohort (Figure 7F).

\section{Discussion}

The dramatic success of immune checkpoint blockade in a variety of notoriously difficult-to-treat cancers has nearly standardized immunomodulation as an approach for cancer treatment (40-42). However, subsequent research has advanced our understanding of how cancer type- and tumor cell-specific characteristics, such as genomic mutational burden, can predict response to immunotherapy $(33,34)$. It has also recently been shown that the state of the immune microenvironment can prevent response to immunotherapy if not appropriately addressed (43). In fact, it has become clear that the complex relationship between cancer cell-intrinsic factors and the subtleties of the cancer type-specific immune microenvironment may make a generalized strategy of immune checkpoint blockade ineffective (44). Therefore, it has become increasingly evident that cancer-specific immunotherapeutic strategies are needed.

In this study, we performed RNA-Seq on 75 human GIST specimens from 75 patients to characterize the immune landscape of different GIST mutational drivers, which we believe is the largest cohort of GISTs to be analyzed with next-generation RNA-Seq. After observing potential differences in immune infiltration, we focused on the 2 prevalent GIST mutational subtypes, namely KIT and PDGFRA, and found that PDGFRA-mutant GISTs contain more immune cells than KIT-mutant GISTs. This suggests that patients with PDGFRA-mutant GIST may ultimately have a greater potential to respond to immunotherapy.

Through differential gene expression analysis, we identified mutation-specific immune landscapes that may be significant for directing GIST immunotherapy. First, PDGFRA-mutant GISTs showed higher expression of CD96, suggesting that NK cells may play a more substantial role in the immune environment of PDGFRA-mutant compared with KIT-mutant GIST. In fact, NK cell infiltrate has been suggested to be a contributor to long-term survival in KIT-mutant GIST (45), which may help explain why PDGFRA-mutant GIST patients have a better over- 
A All KIT vs. all PDGFRA model

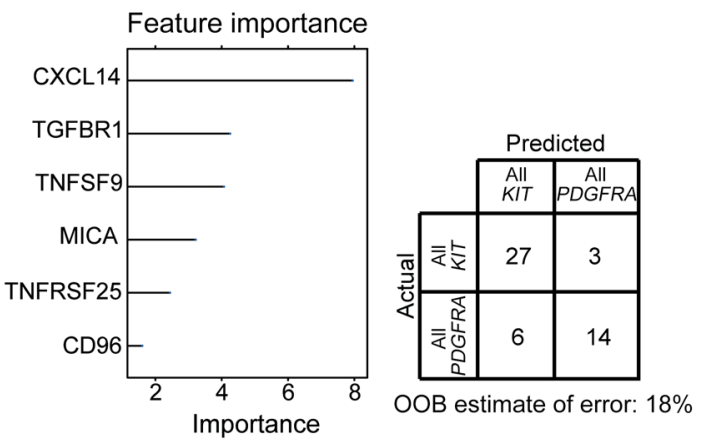

B Distribution of important features

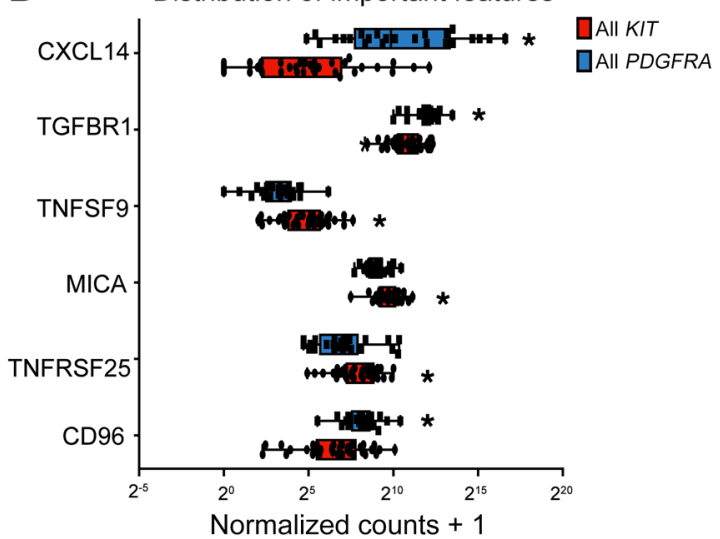

C

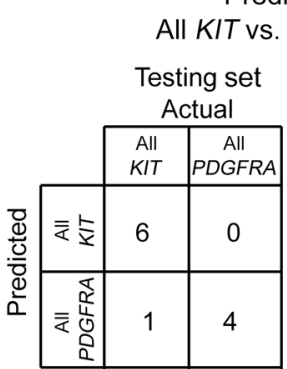

Accuracy: $90.9 \%$ 95\% Cl: [58.7-99.7] $\mathrm{NIR}=63.6 \%$

$P$ value $[$ Acc $>$ NIR]: 0.05

Sensitivity: $100 \%$

Specificity: $85.7 \%$

D UPG KIT vs. UPG PDGFRA model

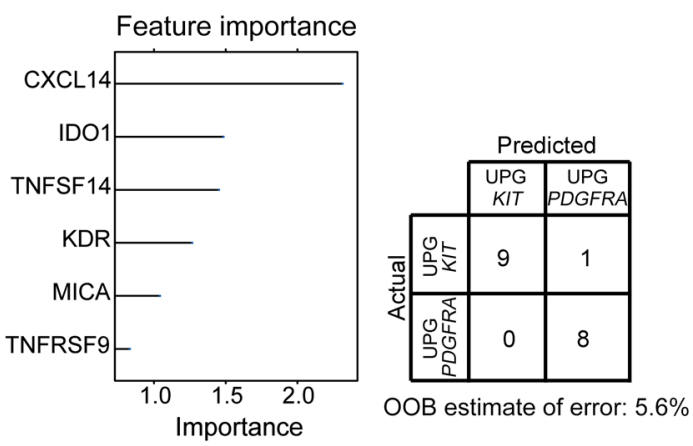

E Distribution of important features

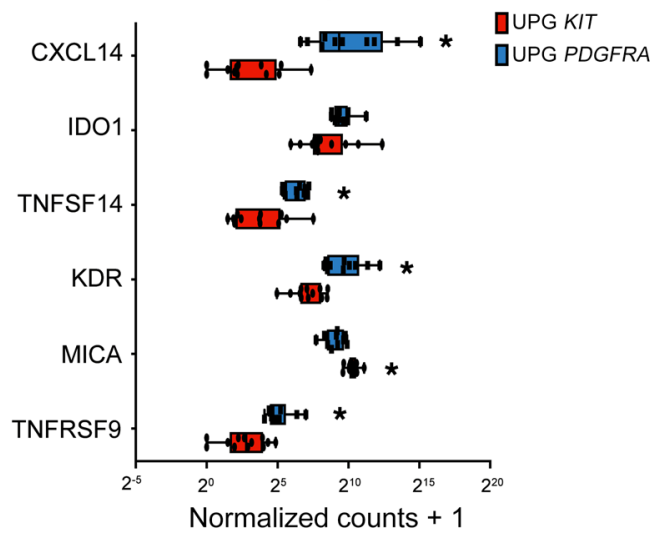

$\mathbf{F}$

Predictive capacity UPG KIT vs. UPG PDGFRA model

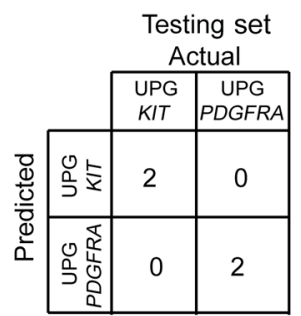

Accuracy: $100 \%$ 95\% Cl: [39.7-100] $\mathrm{NIR}=50 \%$ $P$ value: 0.06 Sensitivity: $100 \%$ Specificity: $100 \%$

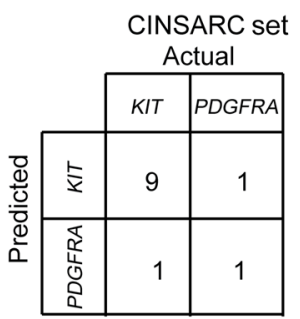

Accuracy: $83.3 \%$ 95\% Cl: [51.6-97.9] NIR $=83.3 \%$ $P$ value: 0.67 Sensitivity: $50 \%$ Specificity $90 \%$ :

Figure 6. Machine learning identifies an immune signature predictive of KIT- and PDCFRA-mutant GIST. (A) Random forest modeling with 5-fold cross-validation of KIT- and PDGFRA-mutant GIST specimens (training set created by partitioning $80 \%$ of $K I T$ and PDCFRA samples from Supplemental Table 3, $n=50$ ). Confusion matrix (right) indicates assessment of model fit to training set. OOB, out-of-bag. (B) Distribution of top 6 features identified by random forest modeling. ${ }^{*}$ Adjusted $P<0.05$ from DSeq2. (C) Predictive capacity of model on remaining KIT- and PDGFRA-mutant GIST testing set $(n=11)$ and the CINSARC cohort ( $n=12)$. Accuracy (Acc), sensitivity, specificity, and $P$ value[Acc $>$ no information rate (NIR)] of the model are shown, calculated by caret package for $R$. Bars indicate mean \pm SEM. (D) Random forest modeling with 5-fold cross-validation of UPG KIT- and UPG PDGFRA-mutant GIST specimens (training set created by partitioning $80 \%$ of UPG KIT and UPG PDGFRA samples from Supplemental Table 4, $n=18$ ). Confusion matrix (right) indicates assessment of model fit to training set. (E) Distribution of top 6 features identified by random forest modeling. *Adjusted $P<0.05$ from DSeq2. (F) Predictive capacity of model on remaining UPG KIT- and UPG PDCFRA-mutant GIST testing set $(n=4)$ and the CINSARC cohort $(n=12)$. Accuracy, sensitivity, specificity, and $P$ value[Acc $>$ NIR] of the model are shown, calculated by caret package for R. For $\mathbf{B}$ and $\mathbf{E}$, all data points are shown, with boxes defining the interquartile range and whiskers extending to the lowest and highest data points.

all prognosis (4). Similarly, PDGFRA-mutant GISTs had higher CD4, CCR4, and CCR5 expression (indicative of regulatory $\mathrm{T}$ cells) and higher expression of the immunomodulators BTLA, CD48, TNFRSF9, and TIGIT. PDGFRA-mutant GISTs, there- fore, may be more receptive to a variety of immunotherapeutic approaches, such as targeting TIGIT, BTLA, CD48, or TNFRSF9 in addition to regulatory $\mathrm{T}$ cells and NK cells, and PD-1/PD-L1 checkpoint blockade. 
A
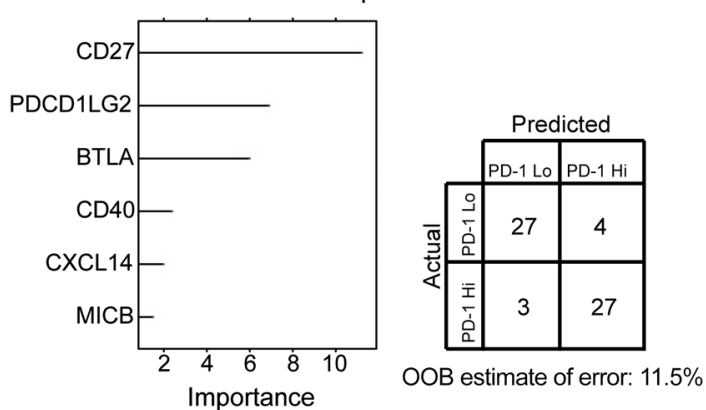

B

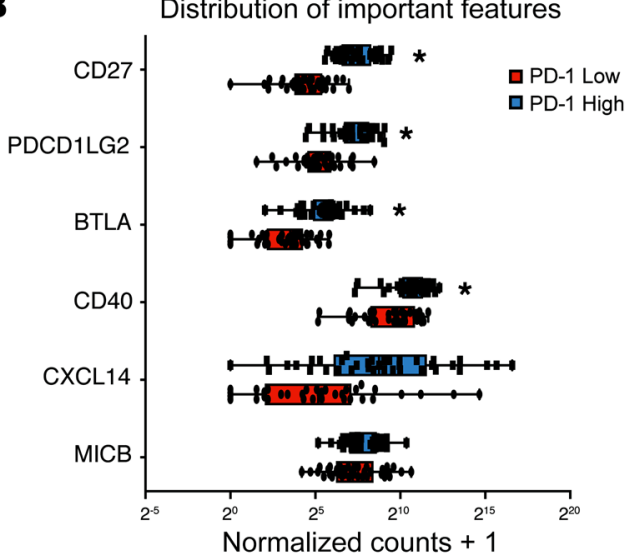

C

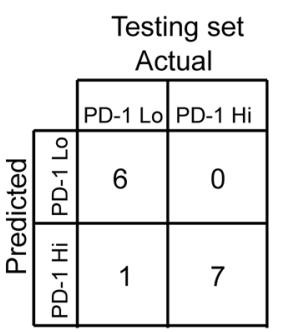

Accuracy: $92.9 \%$ 95\% Cl: [66.1-99.8] $\mathrm{NIR}=50 \%$ $P$ value $\left[\right.$ Acc $>$ NIR] $: 9.2 e^{-4}$ Sensitivity: $100 \%$ Specificity: $85.7 \%$
D

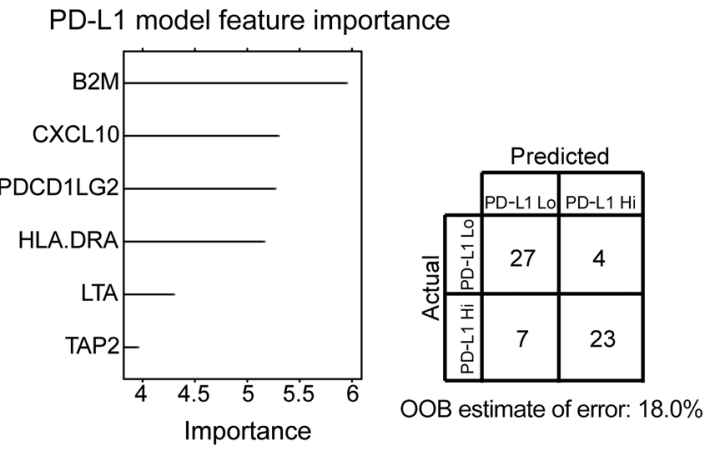

E

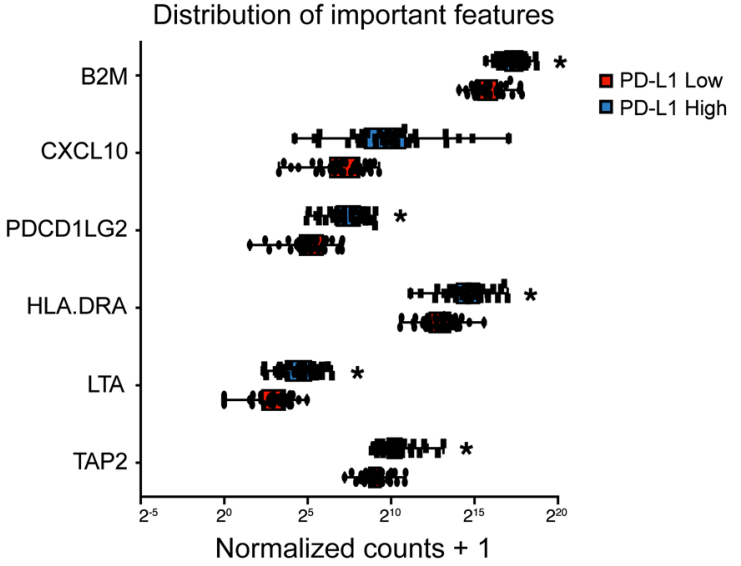

F Predictive capacity of PD-L1 model

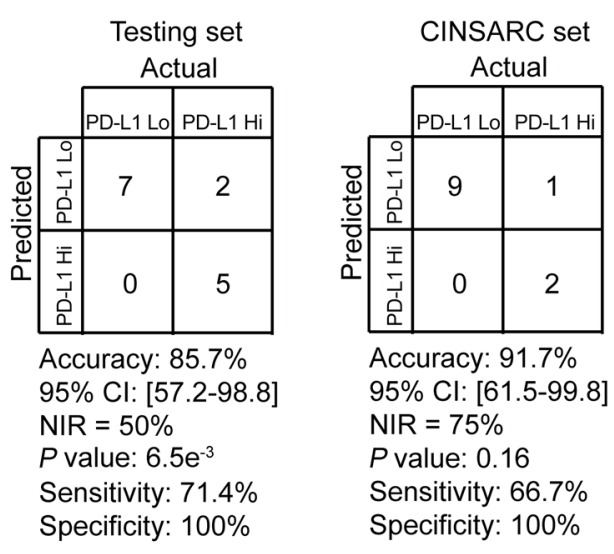

Figure 7. Machine learning identifies an immune signature predictive of PD-1 and PD-L1 expression in GIST. (A) Random forest modeling of PD-1 with 5-fold cross-validation of GIST specimens (training set created by partitioning $80 \%$ of all GIST samples from Supplemental Table $1, n=61$ ). Confusion matrix (right) indicates assessment of model fit to training set. (B) Distribution of top 6 features identified by random forest modeling. ${ }^{*}$ Adjusted $q<0.1$. (C) Predictive capacity of model on remaining 14 GISTs (testing set) and external CINSARC GIST cohort $(n=12)$. Accuracy, sensitivity, specificity, and $P$ value[Acc $>$ NIR] of model are shown, calculated by caret package for R. (D) Random forest modeling of PD-L1 with $5 \mathrm{k}$-folds cross-validation of GIST specimens (training set created by partitioning $80 \%$ of all GIST samples from Supplemental Table $1, n=61$ ). Confusion matrix (right) indicates assessment of modeling fit to training set. (E) Distribution of top 6 features identified by random forest modeling. ${ }^{*}$ Adjusted $q<0.1$. (F) Predictive capacity of model on remaining 14 GISTs (testing set) and external CINSARC GIST cohort $(n=12)$. Accuracy, sensitivity, specificity, and $P$ value[Acc $>$ NIR] of the model are shown, as calculated by caret package for R. For $\mathbf{B}$ and $\mathbf{E}$, all data points are shown, with boxes defining the interquartile range and whiskers extending to the lowest and highest data points.

Comparison of UPG GISTs of the KIT- and PDGFRA-mutant phenotypes revealed that mitotic rate, an indicator of GIST aggressiveness, was inversely related to the quantity of the immune infiltrate. While this concept has been described in other cancers, tumor proliferative index has not yet been shown to affect the quantity of the immune infiltrate in GIST. PDGFRA-mutant GIST has been shown to have a lower proliferative index when compared with KIT-mutant GIST (46). However, when controlling for mutational driver, the relationship between mitotic rate and immune cell infiltration in UPG GISTs loses significance, suggesting that oncogenic driver may be more important for dictating the immune infiltrate. In fact, comparison of UPG KIT- and PDGFRA-mutant 
GISTs revealed distinct cytokine profiles, which we hypothesize may contribute to immune cell chemotaxis. CXCL14 is a relatively recently identified chemokine with a clear role in immune cell attraction $(31,32,47)$. CXCL14 was found to be significantly upregulated in PDGFRA-mutant compared with KIT-mutant GISTs, not only in our cohort but also in a previously published cohort (30). Unfortunately, studying this correlation has proved challenging, as there is currently no PDGFRA-mutant GIST mouse model or human PDGFRA-mutant GIST cell line in which to explore what appears to be a clear biological relationship between PDGFRA tyrosine kinase activation and CXCL14 secretion. Thus, the implication of our finding at this time remains unclear.

It is clear that in cancers with a high number of mutations, tumor mutational burden correlates with immunotherapy response (33). However, the role of neoepitopes in the immune response of cancers with a single oncogenic mutation (i.e., GIST) has not been described. To our surprise, nearly every GIST mutation in our cohort produced a neoepitope capable of binding with high affinity to patient-specific HLA class I, suggesting that even cancers with one mutation can generate a neoepitope recognizable by the immune system. We discovered that the $D 842 \mathrm{~V}$ mutation produced 6 different high-affinity neoepitopes, which bound to 12 different HLA types of patients in this cohort alone, one of which is the most common HLA type in the United States and in White individuals $(35,36)$. While we have not shown that these neoepitopes produced an immune response in these patients, it is intriguing that GISTs with a larger immune infiltrate appear to have a mutation that generates peptides with broad HLA binding specificity, suggesting that this mutation may be of higher quality (37). Moreover, the HLA-specific binding affinities of neoepitopes generated by mutation-specific GIST subtypes reported in this study may provide a road map for patient selection in future neoantigen vaccination trials.

We employed machine learning in order to identify key differences and to profile the expression of immune-related genes between PDGFRA- and KIT-mutant GISTs. Generating a gene expression-based immune profile that accurately predicted the KIT- or PDGFRA-mutant genotype not only supports our hypothesis that the genotype may impact the immune infiltrate, but also introduces another method by which profiling the immune landscapes of KIT- and PDGFRA-mutant tumors can lead to more personalized cancer immunotherapy. Not surprisingly, CXCL14 appeared to be a top feature of PDGFRA-mutant GIST, again suggesting that CXCL14 expression is oncogene specific and may be responsible for the observed differences in immune infiltration and activity. TGFBR1, TNFSF9, MICA, TNFRSF25, CD96, IDO, TNFSF14, KDR, and TNFRSF9 all contributed to the predictive capacity of the immune profiling models, which resulted in $>90 \%$ diagnostic accuracy in our testing set and in $83 \%$ of the CINSARC validation set. We have previously shown the importance of IDO in dictating the immune phenotype of GIST (13). The role of NK cells and the additional immune modulatory receptors identified in this study should continue to be explored.

Since immunotherapy has not yet shown efficacy in advanced and metastatic GIST (48), we sought to explore the immune profiles of PD-1-high and PD-L1-high GISTs across our entire GIST cohort to identify both therapeutic opportunities and potential bar- riers to effective immunotherapy. CD27 was found to be an important feature predictive of high PD-1 expression in our random forest model, which correctly identified high PD-1 expression in $93 \%$ of our testing GIST cohort. Agonistic CD27 antibodies already exist and have shown efficacy with anti-PD-1 in other models, suggesting that this may be an effective strategy for GIST (49). Similarly, the costimulatory and checkpoint receptors CD40 and BTLA, along with the immune checkpoint ligand PDCD1LG2 and the MHC class I protein MICB were features predictive of high PD-1 expression. We have previously shown that CD40 ligation enhances antitumor immunity in our Kit exon 11 mouse model (17), and these other receptors should be considered in future trials.

Notably, applying our PD-1 model to the external CINSARC cohort resulted in only $67 \%$ accuracy, even though our PD-L1 model, in which the same methodology was used, was more accurate (92\%). One reason may be related to the small sample size of the external cohort. However, these models identify high-priority targets for further experimental validation.

Finally, expression of key antigen-presenting machinery including B2M, PDCD1LG2, HLA-DRA, and TAP was found to be highly predictive of PD-L1 expression not only in our cohort $(86 \%$ accuracy) but also in the external CINSARC GIST cohort $(92 \%$ accuracy), suggesting a tumor cell-specific mechanism of linking neoepitope presentation with immune suppression. A similar finding has been observed in HIV-infected cells, where MHC class I machinery was upregulated concurrently with PD-L1 (50). This is an important concept to consider, as efforts to block PD-L1 may result in tumor cell changes that also downregulate MHC class I antigen presentation, which is essential for the $\mathrm{CD} 8^{+} \mathrm{T}$ cell-mediated immune response. High PD-L1 expression has also recently been shown to independently correlate with a worse prognosis in soft tissue sarcomas, which was attributed to an increased degree of immune exhaustion, suppression, and negative regulation (51). In contrast, efforts to enhance antigen presentation using neoantigen peptide vaccination or GM-CSF-expressing tumor cell vaccines may require PD-L1 inhibition.

Ultimately, it is becoming increasingly evident that immunotherapy requires a specific approach targeted to not only the tumor type but also the tumor-specific immune microenvironment. Through RNA-Seq and 4 trained machine learning models, we have characterized important and consistent differences in the immune landscape of GISTs driven by different oncogenic mutations, which we believe may help guide future immunotherapy trials in GIST.

\section{Methods}

Human GIST specimens and clinicopathologic features. Fresh surgical specimens were collected immediately upon resection and flash frozen in liquid nitrogen. RNA was extracted using the RNeasy kit (QIAGEN) as per the kit-specified protocol. Clinicopathologic features were obtained via chart review of patient and pathologic records.

Bioinformatics. Poly(A) selection and next-generation RNA-Seq were performed on an Illumina HiSeq 2500 platform by the MSKCC Integrated Genomics Operations core facility. A minimum of 40-50 million 50-bp paired-end reads were obtained for each sample. RNASeq reads were processed using Trimmomatic v0.36 (52) using the following parameters recommended for paired-end Illumina sequenc- 
ing: PE - phred33, ILLUMINACLIP: TruSeq3-PE.fa:2:30:10, LEADING: 3, TRAILING: 3, SLIDINGWINDOW: 4:15, and MINLEN: 36. Sequencing reads were then aligned to the human genome (version hg38_r88), and gene-level counts were calculated using STAR version 2.6.0a (53). Read counts were then normalized, and DGE analyses were performed on indicated groups using the $\mathrm{R}$ software package DESeq2 $(54,55)$. GSEA was performed using the java GSEA software package (version 3.0) and Molecular Signatures Database (MSigDB) version 6.2 (Broad Institute) $(56,57)$.

The activity of cytotoxic $\mathrm{T}$ cells within each sample was estimated utilizing the CYT score, which was calculated from the geometric mean of the normalized read counts for perforin (PRF1) and granzyme A (GZMA) as previously described (23). The immune cell compartment of these samples was further quantified from the normalized read counts using the $\mathrm{R}$ package ESTIMATE (23). The ESTIMATE algorithm is composed of a nonimmune "stromal score" parameter and an "immune score" parameter. In this work, we refer to ESTIMATE score as the immune score subcomponent of the ESTIMATE algorithm, which infers the degree of immune cell infiltration within tumor tissue based on expression data of 141 immune-related genes previously shown to correlate with the presence of leukocytes (24). The absolute quantities of individual immune cell subtypes was inferred from the normalized read counts by using the CIBERSORT program (29), which employs an externally validated leukocyte gene signature matrix (LM22) of 547 genes designed to distinguish 22 cell phenotypes including $7 \mathrm{~T}$ cell types, B cells, macrophages, monocytes, and NK cells (29). Heatmaps of gene expression data with corresponding clinicopathologic characteristics were created with the $\mathrm{R}$ package ComplexHeatmap (58). ssGSEAs were performed using the $\mathrm{R}$ software package GSVA (59).

Neoepitopes and HLA prediction. Patient HLA haplotypes were inferred using the program seq2HLA (60). For neoepitope identification, the amino acid sequences of patient-specific mutations were mapped to combinations of 8-, 9-, and 10-mer peptides, with the mutation evaluated at each amino acid position. Each patient-specific peptide was then tested with the respective patient-specific HLA type using NetMHCPan 3.0 to identify potential high-affinity neoepitopes $(61,62)$. Neoepitope burden was defined as any epitope with a predicted binding affinity within the top $2 \%$ when compared with 400 random natural peptides, as recommended by NetMHCPan 3.0. High-affinity neoepitopes were further filtered using a binding affinity threshold of $500 \mathrm{nM}$, while very high-affinity neoepitopes were defined as having $<150 \mathrm{nM}$ binding affinity. Finally, to explore mutation-specific neoepitope binding diversity, we tested mutation-specific neoepitopes more broadly against all validated NetMHCPan 3.0 HLA types and visualized them using heatmap. 2 in R.

Machine learning. Random forest modeling with 5-fold cross-validation was performed on the training set using the software package caret for R to identify top predictors (38). One hundred and seventeen features, comprising normalized read counts of various immune genes, and bioinformatically inferred CIBERSORT scores were used to train the model (Supplemental Table 5). For characterization of PDGFRA-specific immune features, KIT- and PDGFRA-mutant GIST samples were first partitioned randomly into training (80\% of samples) and testing sets (20\% of samples). The training data set was used to train a random forest machine learning classifier using 5 -fold cross-validation. During each of the 5 folds, a random forest model was trained on $80 \%$ of the training data, and training accuracy, sensitivity, and specificity were calculated; the remaining $20 \%$ of the training data were then used to calculate testing accuracy, sensitivity, and specificity. Finally, our trained machine learning model was applied to the $20 \%$ testing set and to the external validation CINSARC cohort $(n=12$; ref. 39) in order to assess model accuracy on a naive sample set.

Given the total sample size of our cohort and to prevent overfitting of our model, random forest modeling with 5 -fold cross-validation was re-performed on the training set using only the top 6 identified features, and the model was then applied to the naive testing set and CINSARC external data set to assess for model accuracy. Feature importance is a variable calculated by the caret package, with a higher number indicating that the feature is more important for model accuracy (38). The confusion matrices shown in Figures 6 and 7 reflect the model, with the highest accuracy after 5 -fold cross-validation and optimization by caret. The $P$ value used by caret is calculated using an exact binomial test in which a 1-sided test is performed to determine whether the model accuracy rate is better than the "no information rate," which represents the proportion of data within the majority class. For generation of PD-1- and PD-L1-specific immune features, the same approach was used, with groups split into high and low expression based on median PD-1 (median: 14.1) or PD-L1 (median: 209.5) normalized read counts in the training set.

$q R T-P C R$. Total RNA was extracted from snap-frozen human tumors, reverse transcribed, and amplified with PCR TaqMan probe for CXCL14 (Hs01557413_m1). qRT-PCR was performed using a ViiA 7 real-time PCR system (Applied Biosystems). Data were calculated by the $2^{-\Delta \Delta \mathrm{Ct}}$ method as described in the manufacturer's protocol and were expressed as fold increase over the GIST-T1 or GIST-882 cell line control $(63,64)$.

Flow cytometry. Flow cytometric analysis was immediately performed at the time of specimen collection on freshly obtained human GIST specimens after tumor dissociation as previously described (15). Cells were analyzed on a BD FACSAria or LSRFortessa (BD Biosciences). A human-specific antibody for CD45 (2D1) was obtained from BD Biosciences.

Western blot analysis. Protein from flash-frozen GIST tissue or cell lines was analyzed as described previously (18). Antibodies used against GAPDH (clone D16H11) and PD-L1 (clone E1L3N) were purchased from Cell Signaling Technology.

Histology. Freshly collected tumors were fixed in $4 \%$ paraformaldehyde, embedded in paraffin, and cut into 5- $\mu$ m sections. CD45 (PD7/26 + 2B11) and CD8 (SP57) staining was performed by the MSKCC Molecular Cytology Core. Slides were scanned with MIRAX SCAN (Zeiss) and photographed with Pannoramic Viewer (3DHISTECH Ltd.). For quantification of CD45 and CD8 staining, cell counting was performed manually in a blinded fashion at $\times 20$ magnification (HPF). The number of $\mathrm{CD}^{4} 5^{+}$and $\mathrm{CD} 8^{+}$cells per $\mathrm{HPF}$ was calculated by examining 5 HPFs per tumor and plotting the average per tumor.

Statistics. Some data and graphs were created using Prism 7.0 (Figure 2, A, B, and E; Figure 4, C and D; Figure 5, A and B; Figure 6, B and E; Figure 7, B and E; GraphPad Software). Statistical tests were performed as described in the figure legends, and when possible, adjusted $P$ values calculated by DESeq 2 were used to assess for significance, with a threshold of adjusted $P<0.05$. For Figure 7, B and E, an FDR approach was taken using the Benjamini, Krieger, and Yekutieli 2-stage step-up method, with a $q$ value threshold of $<0.1$ in order to assess for significance. 
Data availability and CINSARC external validation GIST cohort. RNA-Seq data presented in this work are available through the Sequencing Read Archive under accession no. PRJNA521803. RNASeq data of fresh-frozen GIST tumors from the publicly available CINSARC cohort (39) were obtained from the Sequence Read Archive under accession no. SRP057793.

Study approval. Tumor specimens were obtained from 2011 to 2017 from 75 GIST patients who underwent surgical resection at MSKCC. Prior to resection, patients provided written informed consent under an IRB-approved protocol. Using a prospectively maintained institutional database, clinicopathologic characteristics were documented and encrypted, and all research was conducted in compliance with Health Insurance Portability and Accountability Act regulations.

\section{Author contributions}

GAV, TGB, BDM, and RPD contributed to study conception and design. GAV, TGB, and RPD contributed to the development of research methodology. GAV, TGB, BDM, JQZ, JKL, NJP, SZ, ML, and $\mathrm{FC}$ contributed to the acquisition of data and conduction of experiments. GAV, TGB, ML, RLG, NJP, FR, and RPD contributed to the analysis and interpretation of data. GAV, TGB, and RPD contributed to the writing, review, and revision of the manuscript. All authors reviewed and contributed comments that were incorporated into the final version of this manuscript. Study supervision was provided by RPD.

\section{Acknowledgments}

The investigators were supported by NIH grants R01 CA102613 and T32 CA009501, The David Foundation, Betsy Levine-Brown and Marc Brown, David and Monica Gorin, and the GIST Cancer Research Fund (RPD); and Division of Loan Repayment NIH grant L30 TR002111 (GAV). The Molecular Cytology Core Facility at MSKCC was supported by Cancer Center Support Grant P30 CA008748. We thank Russell Holmes for logistical and administrative support.

Address correspondence to: Ronald P. DeMatteo, 3400 Spruce Street, Philadelphia, Pennsylvania19104,USA.Phone:215.662.7539; Email: ronald.dematteo@uphs.upenn.edu.
1. Ducimetière F, et al. Incidence of sarcoma histotypes and molecular subtypes in a prospective epidemiological study with central pathology review and molecular testing. PLOS ONE. 2011;6(8):e20294.

2. Hirota S, et al. Gain-of-function mutations of c-kit in human gastrointestinal stromal tumors. Science. 1998;279(5350):577-580.

3. Joensuu H, DeMatteo RP. The management of gastrointestinal stromal tumors: a model for targeted and multidisciplinary therapy of malignancy. Annu Rev Med. 2012;63:247-258.

4. Corless CL, et al. Pathologic and molecular features correlate with long-term outcome after adjuvant therapy of resected primary GI stromal tumor: the ACOSOG Z9001 trial. J Clin Oncol. 2014;32(15):1563-1570.

5. Corless CL, et al. PDGFRA mutations in gastrointestinal stromal tumors: frequency, spectrum and in vitro sensitivity to imatinib. J Clin Oncol. 2005;23(23):5357-5364.

6. Lasota J, Dansonka-Mieszkowska A, Sobin LH, Miettinen M. A great majority of GISTs with PDGFRA mutations represent gastric tumors of low or no malignant potential. Lab Invest. 2004;84(7):874-883.

7. Wardelmann E, et al. Association of plateletderived growth factor receptor alpha mutations with gastric primary site and epithelioid or mixed cell morphology in gastrointestinal stromal tumors. J Mol Diagn. 2004;6(3):197-204.

8. Cavnar MJ, et al. Rectal gastrointestinal stromal tumor (GIST) in the era of imatinib: organ preservation and improved oncologic outcome. Ann Surg Oncol. 2017;24(13):3972-3980.

9. Oppelt PJ, Hirbe AC, Van Tine BA. Gastrointestinal stromal tumors (GISTs): point mutations matter in management, a review. J Gastrointest Oncol. 2017;8(3):466-473.

10. Burgoyne AM, et al. Duodenal-jejunal flexure GI stromal tumor frequently heralds somatic NF1 and Notch pathway mutations. JCO Precis Oncol.
https://doi.org/10.1200/PO.17.00014.

11. Miettinen M, Wang ZF, Sarlomo-Rikala M, Osuch C, Rutkowski P, Lasota J. Succinate dehydrogenase-deficient GISTs: a clinicopathologic, immunohistochemical, and molecular genetic study of 66 gastric GISTs with predilection to young age. Am J Surg Pathol. 2011;35(11):1712-1721.

12. Shi E, et al. FGFR1 and NTRK3 actionable alterations in "wild-type" gastrointestinal stromal tumors. J Transl Med. 2016;14(1):339.

13. Balachandran VP, et al. Imatinib potentiates antitumor $\mathrm{T}$ cell responses in gastrointestinal stromal tumor through the inhibition of Ido. Nat Med. 2011;17(9):1094-1100.

14. Cavnar MJ, et al. KIT oncogene inhibition drives intratumoral macrophage M2 polarization. J Exp Med. 2013;210(13):2873-2886.

15. Seifert AM, et al. PD-1/PD-L1 Blockade enhances T-cell activity and antitumor efficacy of imatinib in gastrointestinal stromal tumors. Clin Cancer Res. 2017;23(2):454-465.

16. Sommer G, et al. Gastrointestinal stromal tumors in a mouse model by targeted mutation of the Kit receptor tyrosine kinase. Proc Natl Acad Sci US A. 2003;100(11):6706-6711.

17. Zhang JQ, et al. Macrophages and CD8+ T cells mediate the antitumor efficacy of combined CD40 ligation and imatinib therapy in gastrointestinal stromal tumors. Cancer Immunol Res. 2018;6(4):434-447.

18. Vitiello GA, et al. Mitochondrial inhibition aug ments the efficacy of imatinib by resetting the metabolic phenotype of gastrointestinal stromal tumor. Clin Cancer Res. 2018;24(4):972-984.

19 . Zeng $\mathrm{S}$, et al. Wnt/ $\beta$-catenin signaling contributes to tumor malignancy and is targetable in gastrointestinal stromal tumor. Mol Cancer Ther. 2017;16(9):1954-1966.

20. Zeng S, et al. ETV4 collaborates with Wnt/ $\beta$-catenin signaling to alter cell cycle activity and promote tumor aggressiveness in gastrointestinal stromal tumor. Oncotarget. 2017;8(69):114195-114209.
21. Lawrence MS, et al. Mutational heterogeneity in cancer and the search for new cancer-associated genes. Nature. 2013;499(7457):214-218.

22. Şenbabaoğlu Y, et al. Tumor immune microenvironment characterization in clear cell renal cell carcinoma identifies prognostic and immunotherapeutically relevant messenger RNA signatures. Genome Biol. 2016;17(1):231.

23. Rooney MS, Shukla SA, Wu CJ, Getz G, Hacohen N. Molecular and genetic properties of tumors associated with local immune cytolytic activity. Cell. 2015;160(1-2):48-61.

24. Yoshihara K, et al. Inferring tumour purity and stromal and immune cell admixture from expression data. Nat Commun. 2013;4:2612.

25. Pantaleo MA, Nannini M, Corless CL, Heinrich MC. Quadruple wild-type (WT) GIST: defining the subset of GIST that lacks abnormalities of KIT, PDGFRA, SDH, or RAS signaling pathways. Cancer Med. 2015;4(1):101-103.

26. Gold JS, et al. Development and validation of a prognostic nomogram for recurrence-free survival after complete surgical resection of localised primary gastrointestinal stromal tumour: a retrospective analysis. Lancet Oncol. 2009;10(11):1045-1052.

27. Cameron S, Gieselmann M, Blaschke M, Ramadori $G$, Füzesi L. Immune cells in primary and metastatic gastrointestinal stromal tumors (GIST). Int J Clin Exp Pathol. 2014;7(7):3563-3579.

28. Charoentong P, et al. Pan-cancer immunogenomic analyses reveal genotype-immunophenotype relationships and predictors of response to checkpoint blockade. Cell Rep. 2017;18(1):248-262.

29. Newman AM, et al. Robust enumeration of cell subsets from tissue expression profiles. Nat Methods. 2015;12(5):453-457.

30. Kang HJ, et al. Correlation of KIT and plateletderived growth factor receptor alpha mutations with gene activation and expression profiles in gastrointestinal stromal tumors. Oncogene. 2005;24(6):1066-1074. 
31. Cicchini L, et al. Suppression of antitumor immune responses by human papillomavirus through epigenetic downregulation of CXCL14. MBio. 2016;7(3):e00270-16.

32. Lee HT, et al. A crucial role of CXCL14 for promoting regulatory $\mathrm{T}$ cells activation in stroke. Theranostics. 2017;7(4):855-875.

33. Goodman AM, et al. Tumor mutational burden as an independent predictor of response to immunotherapy in diverse cancers. Mol Cancer Ther. 2017;16(11):2598-2608.

34. Hellmann MD, et al. Genomic features of response to combination immunotherapy in patients with advanced non-small-cell lung cancer. Cancer Cell. 2018;33(5):843-852.e4.

35. Ellis JM, Henson V, Slack R, Ng J, Hartzman RJ, Katovich Hurley C. Frequencies of HLA-A2 alleles in five U.S. population groups. Predominance $\mathrm{Of}^{*} 02011$ and identification of HLA$\mathrm{A}^{*}$ 0231. Hum Immunol. 2000;61(3):334-340.

36. Maiers M, Gragert L, Klitz W. High-resolution HLA alleles and haplotypes in the United States population. Hum Immunol. 2007;68(9):779-788.

37. Balachandran VP, et al. Identification of unique neoantigen qualities in long-term survivors of pancreatic cancer. Nature. 2017;551(7681):512-516.

38. Kuhn M. Building predictive models in R using the caret package. Journal of Statistical Software Web site. https://www.jstatsoft.org/article/ view/v028i05. Published November 10, 2008. Accessed February 14, 2019.

39. Lesluyes $\mathrm{T}$, et al. RNA sequencing validation of the Complexity INdex in SARComas prognostic signature. Eur J Cancer. 2016;57:104-111.

40. Couzin-Frankel J. Breakthrough of the year 2013. Cancer immunotherapy. Science. 2013;342(6165):1432-1433.

41. Topalian SL, et al. Safety, activity, and immune correlates of anti-PD-1 antibody in cancer. N Engl JMed. 2012;366(26):2443-2454.

42. Wolchok JD, Rollin L, Larkin J. Nivolumab and
Ipilimumab in Advanced Melanoma. $N$ Engl Med. 2017;377(25):2503-2504.

43. Messenheimer DJ, et al. Timing of PD- 1 blockade is critical to effective combination immunotherapy with anti-OX40. Clin Cancer Res. 2017;23(20):6165-6177.

44. Topalian SL, Drake CG, Pardoll DM. Immune checkpoint blockade: a common denominator approach to cancer therapy. Cancer Cell. 2015;27(4):450-461.

45. Ménard C, et al. Natural killer cell IFN-gamma levels predict long-term survival with imatinib mesylate therapy in gastrointestinal stromal tumor-bearing patients. Cancer Res. 2009;69(8):3563-3569.

46. Campanella NC, et al. Lack of microsatellite instability in gastrointestinal stromal tumors. Oncol Lett. 2017;14(5):5221-5228.

47. Lu J, Chatterjee M, Schmid H, Beck S, Gawaz M. CXCL14 as an emerging immune and inflammatory modulator. J Inflamm (Lond). 2016;13:1.

48. D'Angelo SP, et al. Combined KIT and CTLA-4 blockade in patients with refractory GIST and other advanced sarcomas: a phase Ib study of dasatinib plus ipilimumab. Clin Cancer Res. 2017;23(12):2972-2980.

49. Buchan SL, et al. PD-1 Blockade and CD27 stimulation activate distinct transcriptional programs that synergize for CD8+ T-cell-driven antitumor immunity. Clin Cancer Res. 2018;24(10):2383-2394.

50. Pai SI, et al. HLA class I antigen processing machinery (APM) component expression and PD-1:PD-L1 pathway activation in HIV-infected head and neck cancers. Oral Oncol. 2018;77:92-97.

51. Bertucci F, et al. PDL1 expression is a poor-prognosis factor in soft-tissue sarcomas. Oncoimmunology. 2017;6(3):e1278100.

52. Bolger AM, Lohse M, Usadel B. Trimmomatic: a flexible trimmer for Illumina sequence data. Bioinformatics. 2014;30(15):2114-2120.

53. Dobin A, et al. STAR: ultrafast universal RNA-seq aligner. Bioinformatics. 2013;29(1):15-21.

54. Love MI, Huber W, Anders S. Moderated estimation of fold change and dispersion for RNA-seq data with DESeq2. Genome Biol. 2014;15(12):550.

55. R Core Team. R: a language and environment for statistical computing. R Foundation for Statistical Computing, Vienna, Austria. https:// www.r-project.org/. Accessed February 14, 2019.

56. Mootha VK, et al. PGC-1alpha-responsive genes involved in oxidative phosphorylation are coordinately downregulated in human diabetes. Nat Genet. 2003;34(3):267-273.

57. Subramanian A, et al. Gene set enrichment analysis: a knowledge-based approach for interpreting genome-wide expression profiles. Proc Natl Acad Sci US A. 2005;102(43):15545-15550.

58. Gu Z, Eils R, Schlesner M. Complex heatmaps reveal patterns and correlations in multidimensional genomic data. Bioinformatics. 2016;32(18):2847-2849.

59. Hänzelmann S, Castelo R, Guinney J. GSVA: gene set variation analysis for microarray and RNAseq data. BMC Bioinformatics. 2013;14:7.

60. Boegel S, et al. HLA typing from RNA-Seq sequence reads. Genome Med. 2012;4(12):102.

61. Hoof I, et al. NetMHCpan, a method for MHC class I binding prediction beyond humans. Immunogenetics. 2009;61(1):1-13.

62. Nielsen M, Andreatta M. NetMHCpan-3.0; improved prediction of binding to MHC class I molecules integrating information from multiple receptor and peptide length datasets. Genome Med. 2016;8(1):33.

63. Taguchi $\mathrm{T}$, et al. Conventional and molecular cytogenetic characterization of a new human cell line, GIST-T1, established from gastrointestinal stromal tumor. Lab Invest. 2002;82(5):663-665.

64. Tuveson DA, et al. STI571 inactivation of the gastrointestinal stromal tumor c-KIT oncoprotein: biological and clinical implications. Oncogene. 2001;20(36):5054-5058 